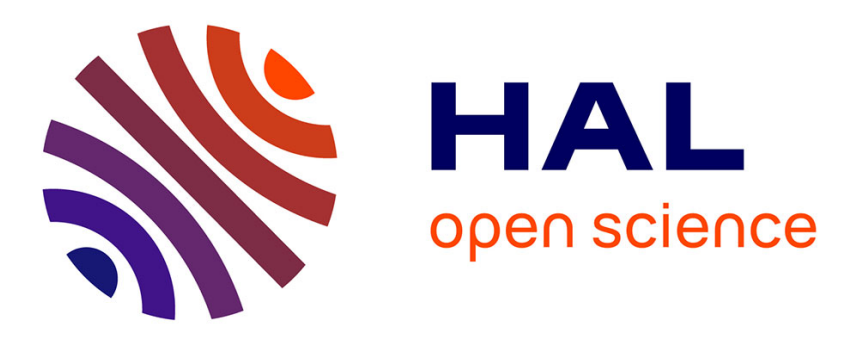

\title{
How to minimally modify a dynamical system when constructing flat inputs?
}

Florentina Nicolau, Witold Respondek, Jean-Pierre Barbot

\section{To cite this version:}

Florentina Nicolau, Witold Respondek, Jean-Pierre Barbot. How to minimally modify a dynamical system when constructing flat inputs?. International Journal of Robust and Nonlinear Control, In press, $10.1002 /$ rnc.5790 . hal-02933584v2

\section{HAL Id: hal-02933584 \\ https://hal.science/hal-02933584v2}

Submitted on 10 Sep 2021

HAL is a multi-disciplinary open access archive for the deposit and dissemination of scientific research documents, whether they are published or not. The documents may come from teaching and research institutions in France or abroad, or from public or private research centers.
L'archive ouverte pluridisciplinaire HAL, est destinée au dépôt et à la diffusion de documents scientifiques de niveau recherche, publiés ou non, émanant des établissements d'enseignement et de recherche français ou étrangers, des laboratoires publics ou privés. 


\title{
How to minimally modify a dynamical system when constructing flat inputs?
}

\author{
Florentina Nicolau*, Witold Respondek $\nmid$ and Jean-Pierre Barbot $\ddagger$
}

\begin{abstract}
In this paper, we study the problem of constructing flat inputs for multi-output dynamical systems, in particular, we address the issue of the minimal modification of the initial dynamical system (the measure of modification being the number of equations that have to be changed by adding flat inputs). We show that in the observable case, control vector fields that distort $m$ equations only (where $m$ is the number of measurements) can always be constructed (and this is the minimal possible number of equations that have to be modified by adding flat inputs), while in the unobservable case, the best that we can hope for is that $m+1$ equations only are modified such that they involve flat inputs. We discuss when the original output is a flat output of minimal possible differential weight for the minimally modified control system (where by the differential weight, we mean the minimal number of derivatives of components of a flat output needed to express all states and controls). We propose a solution for constructing flat inputs leading to a minimally modified control system consistent with the minimal differential weight and, moreover, for which the observable part is affected by the minimal possible number of controls (this last property being important in applications). We show that, in that case, at least $2 m-1$ equations have to be affected by the flat inputs.
\end{abstract}

Keywords: flat inputs, flatness, observed dynamical systems, minimal modification, constructing control vector fields.

\section{Introduction}

Flat inputs have been introduced by Waldherr and Zeitz [32, 33] and are objects dual to flat outputs. Their construction can been seen as a dual problem to that of constructing a flat output. More precisely, given a nonlinear observed dynamics of the form:

$$
\Sigma: \dot{x}=f(x), \quad y=h(x),
$$

with $x \in \mathbb{X}$, an $n$-dimensional manifold (in particular, $\mathbb{X}=\mathbb{R}^{n}$ or $\mathbb{X}$ is an open subset of $\mathbb{R}^{n}$ ) and $y \in \mathbb{R}^{m}$ the measurements (supposed independent everywhere), the problem of

${ }^{*}$ QUARTZ EA7393 Laboratory, ENSEA, 6 Avenue du Ponceau, 95014 Cergy-Pontoise, France. Currently in secondment at L2S CNRS UMR 8506, CentraleSupélec, Gif-sur-Yvette, France. florentina.nicolau@ensea.fr

${ }^{\dagger}$ Normandie Université, INSA de Rouen, Laboratoire de Mathématiques EA 3226 - FR CNRS 3335, 76801 Saint-Etienne-du-Rouvray, France. witold.respondek@insa-rouen.fr

${ }^{\ddagger}$ QUARTZ EA7393 Laboratory, ENSEA, 6 Avenue du Ponceau, 95014 Cergy-Pontoise, France. Currently in secondment at LS2N-CNRS UMR 6004, 44322 Nantes, France. Currently in secondment at LS2N CNRS UMR 6004, E.C.N, Nantes, France. barbot@ensea.fr 
constructing flat inputs consists in finding control vector fields $g_{1}, \ldots, g_{m}$ (or equivalently, to place the actuators or the inputs) such that the control-affine system

$$
\Sigma_{c}: \dot{x}=f(x)+\sum_{i=1}^{m} g_{i}(x) u_{i},
$$

associated to $\Sigma$, is flat with the original measurements $\left(h_{1}, \ldots, h_{m}\right)$ being a flat output. The vector fields $f, g_{i}$ and the functions $h_{i}$, for $1 \leq i \leq m$, are supposed $\mathcal{C}^{\infty}$-smooth. The inputs $u_{1}, \ldots, u_{m}$ multiplying, resp., $g_{1}, \ldots, g_{m}$, are called in [32, 33] flat inputs. The single-output case has been considered in [32], while the observable multi-output case has been discussed in [33], see also [7] for another approach based on the notion of unimodularity. In the recent papers [19, 20] (see also [6]), the authors solved the unobservable case for which locally, around any point of an open and dense subset of the state space, we constructed control vector fields $g_{1}, \ldots, g_{m}$ such that the control-affine system $\Sigma_{c}$ is flat with $h$ being a flat output.

One of the motivations to construct a flat input for a given output is that with such an input, the tracking problem for that output can be solved with no need to calculate the zero dynamics (see, e.g., [10, 28]) but constructing flat inputs may be useful for other problems as well, like parameter identification [27] or private communication [21] using chaotic systems (see also [2], for a related approach using the design of nonlinear observers with unknown inputs, and [4, 30, 31], where left invertibility and flatness of switched linear discrete-time systems is applied to private communication and cryptographic applications). Similarly to the construction of a flat output (that can be seen as a problem of sensors placement in order to achieve flatness of the resulting input-stateoutput system) for which, from a technological point of view, it is not always possible to place the sensors exactly where we want them, for constructing flat inputs it may also be technologically difficult to place the actuators at the right place. Nevertheless, as mentioned above, the flat inputs approach can be very useful in applications, in the process design of an optimal closed loop feedback control. By adding flat inputs, we change the original dynamics $\dot{x}=f(x)$ of the system and therefore, some of its properties. For some applications, it may be important to modify the original system in a minimal way, namely when certain properties of the original dynamical system have to be preserved for the flat control system. For instance, in the context of private communication, the chaotic behavior of the original dynamics is crucial and has to be preserved by adding the modification $u_{1} g_{1}(x)+\cdots+u_{m} g_{m}(x)$ to $f(x)$. Therefore, it is important to modify the original chaotic system (corresponding to the transmitter dynamics) in a minimal way, see also [15.

A measure of modification that we propose is the number of equations of $\Sigma$ that we have to change by adding flat-inputs, or equivalently, the number of nonzero components of the control vector fields $g_{1}, \ldots, g_{m}$ that render $\left(\Sigma_{c}, h\right)$ flat (each modified equation is supposed to be affected by one flat input only). The control vector fields $g_{1}, \ldots, g_{m}$ have together at least $m$ nonzero components (at least one for each $g_{i}$ ), so at least $m$ equations of the original dynamical system have to be modified to achieve flatness. We show that in the observable case, we can construct vector fields that distort $m$ equations only, while in the nonobservable case, the best that we can hope for (if we require to respect the decomposition into observable and completely unobservable subsystems) is that only $m+1$ equations involve the control.

The main results of the paper are based, similarly to those of [20], on two normal forms $\mathrm{NF}_{\text {min }}$ and $\mathrm{NF}_{\text {min }}^{*}$ (for both $h$ is a flat output) describing the unobservable case. The first normal form $\mathrm{NF}_{\min }$ is minimally modified $(m+1$ equations only are affected by the controls) and only one linking term (that is, only one non-constant component of 
one control vector field is used to render the system observable by linking the originally observed, via the given output $h$, subsystem and the unobserved one) is needed. The differential weight of $h$ (which is the minimal number of time-derivatives of $h_{i}$ 's needed to express all states $x$ and controls $u$ ) as a flat output of $\mathrm{NF}_{\min }$ is however far (except for two very particular cases) from being the minimal possible (and it is always, except for those two cases, greater than that of $h$ as a flat output of the forms provided by [20]). Moreover the original observed (via the given output $h$ ) subsystem is affected by $m-1$ different flat inputs (which is the maximal possible), while the unobserved one is affected by one control only. From the point of view of applications to secure communication, the unobserved part plays the role of a second level of security and we would like it to involve the maximal number of inputs. This leads to the problem of constructing a normal form of, first, minimal differential weight and, second, unobserved part affected by $m-1$ controls. The second result of the paper is a construction of the consistent normal form $\mathrm{NF}_{\text {min }}^{*}$ (where by consistent we mean throughout respecting these two requirements). The form $\mathrm{NF}_{\min }^{*}$ has $2 m-1$ modified equations and we show that for a consistent minimal modification this is the minimal possible. For $\mathrm{NF}_{\min }^{*}$ we need an $(m-1)$-tuple of linking terms (satisfying, as for $\mathrm{NF}_{\min }$, some particular properties).

Both normal forms are local. The nature of the nominal point around which we work (equilibrium or not) plays an important role in our study. If $x_{0}$ is not an equilibrium point of the original system, then $\mathrm{NF}_{\text {min }}$ and $\mathrm{NF}_{\text {min }}^{*}$ can always be constructed as assured by Theorems 3.2 and 3.3 , respectively. Necessary and sufficient conditions for the case of an equilibrium $f\left(x_{0}\right)=0$ are also given in Theorems 3.2 and 3.3 .

Each of the normal forms has its interest. If the goal is to modify the original $\Sigma$ in the simplest possible way (without asking for $h$ to be of minimal differential weight, or imposing any constraints on the number of flat inputs that are added to the observed part), then $\mathrm{NF}_{\text {min }}$ is such a construction. If, on the other hand, it is important to obtain a flat control system minimally modified and simultaneously with the differential weight of $h$ as its flat output being the minimal possible, or with a minimal number of inputs affecting the observed subsystem, then $\mathrm{NF}_{\text {min }}^{*}$ provides such a construction.

The paper is organized as follows. In Section 2, we recall the definitions of flatness and of differential weight of a flat system as well as the notions of observability quasi-indices and partial observability quasi-indices (simply called OQI's, resp., POQI's) introduced in [20] and that will be used in the paper. In Section 3, we give our main results and compare them to those of [20. We deal with the observable case in Theorem 3.1. We propose the local minimally modified normal form $\mathrm{NF}_{\min }$ in Theorem 3.2 and consistent normal form $\mathrm{NF}_{\min }^{*}$ in Theorem 3.3. We provide an example illustrating the differences between $\mathrm{NF}_{\text {min }}$ and $\mathrm{NF}_{\text {min }}^{*}$ at the end of Section 3.2.1. We discuss the problem of a global construction of control vector fields that minimally modify the original dynamics in Section 3.4. Finally, in Section 4, we analyze from the point of view of a minimal modification the solution proposed in [20] for an application to private communication and provide proofs in Section 5 .

\section{Definitions and notations}

Consider a nonlinear control system of the form $\Xi: \dot{x}=f(x)+\sum_{i=1}^{m} g_{i}(x) u_{i}$, where $x \in \mathbb{X}, u \in \mathbb{R}^{m}$ and the vector fields $g_{1}, \ldots, g_{m}$ are independent.

Definition 2.1. The control system $\Xi$ is $x$-flat around $x_{0}$, generically with respect to $u$, if there exist $m$ smooth functions $\varphi_{i}=\varphi_{i}(x), 1 \leq i \leq m$, defined in a neighborhood $\mathcal{O}$ of $x_{0}$, having the following property: there exist an integer $s \geq 1$ and smooth maps 
$(\gamma, \delta): \widetilde{\mathcal{O}} \times \mathbb{R}^{m(s-1)} \rightarrow \mathbb{R}^{n} \times \mathbb{R}^{m}$ such that

$$
x=\gamma\left(\varphi, \dot{\varphi}, \ldots, \varphi^{(s-1)}\right) \text { and } u=\delta\left(\varphi, \dot{\varphi}, \ldots, \varphi^{(s)}\right)
$$

for any $C^{s-1}$-control $u(t)$ and corresponding trajectory $x(t)$ that satisfy $(x(t), u(t)) \in \widetilde{\mathcal{O}}$, where either $\widetilde{\mathcal{O}}=\mathcal{O} \times \mathbb{R}^{m}$ or $\widetilde{\mathcal{O}}$ is a subset of $\mathcal{O} \times \mathbb{R}^{m}$ such that for any $x \in \mathcal{O}$, the set $\{u:(x, u) \in \widetilde{\mathcal{O}}\}$ is open and dense in $\mathbb{R}^{m}$. The $m$-tuple $\varphi=\left(\varphi_{1}, \ldots, \varphi_{m}\right)$ is called a flat output.

It is commonly accepted [5, 16] that flatness is a generic property, that is, even if all functions $\varphi_{i}(x)$ are defined globally, the desired description (2) is local and holds out of singular states and singular values of controls. In the first case, corresponding to $\widetilde{\mathcal{O}}=\mathcal{O} \times \mathbb{R}^{m}$, representation (2) is global with respect to the controls. In the second case, for each $x \in \mathcal{O}$, the set of controls $u$ for which representation (2) holds, is open and dense in $\mathbb{R}^{m}$ (and the pairs $(x, u) \notin \widetilde{\mathcal{O}}$ form singularities of flatness because (2) fails to hold). From now on, when we say that a control system is $x$-flat (or simply flat) at $x_{0}$, we mean that it is $x$-flat at $x_{0}$, generically with respect to $u$ (i.e., satisfies Definition 2.1).

There exists a more general notion of flatness for which the functions $\varphi_{i}$ may depend on the control and its successive time-derivatives up to a certain order $q$, i.e., $\varphi_{i}=$ $\varphi_{i}\left(x, u, \dot{u}, \ldots, u^{(q)}\right)$. We do not need this general notion since, in our study, all functions $\varphi_{i}$ depend on the state $x$ only and singularities depend on $x$ and/or $u$ (but never on derivatives of $u$ ). We send the reader to [8], where a generalized definition of flat inputs is proposed that allows affine injections of the input and its derivatives in both the dynamics and the output equation.

The minimal number of derivatives of components of a flat output, needed to express $x$ and $u$, is called the differential weight of that flat output [18, 25] and is formalized as follows. By definition, for any flat output $\varphi$ of $\Xi$ there exist integers $s_{1}, \ldots, s_{m}$ such that

$$
\begin{aligned}
x & =\gamma\left(\varphi_{1}, \dot{\varphi}_{1}, \ldots, \varphi_{1}^{\left(s_{1}\right)}, \ldots, \varphi_{m}, \dot{\varphi}_{m}, \ldots, \varphi_{m}^{\left(s_{m}\right)}\right) \\
u & =\delta\left(\varphi_{1}, \dot{\varphi}_{1}, \ldots, \varphi_{1}^{\left(s_{1}\right)}, \ldots, \varphi_{m}, \dot{\varphi}_{m}, \ldots, \varphi_{m}^{\left(s_{m}\right)}\right) .
\end{aligned}
$$

Moreover, we can choose $\left(s_{1}, \ldots, s_{m}\right), \gamma$ and $\delta$ such that (see [25]) if for any other $m$-tuple $\left(\tilde{s}_{1}, \ldots, \tilde{s}_{m}\right)$ and functions $\tilde{\gamma}$ and $\tilde{\delta}$, we have

$$
\begin{aligned}
& x=\tilde{\gamma}\left(\varphi_{1}, \dot{\varphi}_{1}, \ldots, \varphi_{1}^{\left(\tilde{s}_{1}\right)}, \ldots, \varphi_{m}, \dot{\varphi}_{m}, \ldots, \varphi_{m}^{\left(\tilde{s}_{m}\right)}\right) \\
& u=\tilde{\delta}\left(\varphi_{1}, \dot{\varphi}_{1}, \ldots, \varphi_{1}^{\left(\tilde{s}_{1}\right)}, \ldots, \varphi_{m}, \dot{\varphi}_{m}, \ldots, \varphi_{m}^{\left(\tilde{s}_{m}\right)}\right),
\end{aligned}
$$

then $s_{i} \leq \tilde{s}_{i}$, for $1 \leq i \leq m$. We will call $\sum_{i=1}^{m}\left(s_{i}+1\right)=m+\sum_{i=1}^{m} s_{i}$ the differential weight of $\varphi$. A flat output of $\Xi$ is called minimal if its differential weight is the lowest among all flat outputs of $\Xi$. The differential weight of a flat system $\Xi$ is equal to the differential weight of a minimal flat output, and is at least $n+m$, since we have to express $n$ states and $m$ independent controls and in order to do that, we need at least $n+m$ derivatives (taking into account also those of order zero).

Flatness is a property of the state-space dynamics $\dot{x}=f(x)+\sum_{i=1}^{m} g_{i}(x) u_{i}$ of a control system. It can also be described as a property of the input-state-output map for a dummy output $y$. In fact, $x$-flatness is equivalent to the existence of an $\mathbb{R}^{m}$-valued dummy output $y=\varphi(x)$ that renders $\dot{x}=f(x)+\sum_{i=1}^{m} g_{i}(x) u_{i}$ observable [13, 14, 34] and left-invertible [24]. Indeed, expressing the state as $x=\gamma\left(\varphi, \dot{\varphi}, \ldots, \varphi^{(s-1)}\right)$ and the control as $u=\delta\left(\varphi, \dot{\varphi}, \ldots, \varphi^{(s)}\right)$ corresponds, resp., to observability and left-invertibility.

Consider the dynamical system $\Sigma$ whose state $x \in \mathbb{X}$, an $n$-dimensional manifold, together with the output $y=h(x) \in \mathbb{R}^{m}$, given by (1). Most of the results of the paper are local, so $\mathbb{X}$ can be simply taken as an open subset of $\mathbb{R}^{n}$. In order to emphasize the 
fact that the system is observed, we will use the notation $(\Sigma, h)$. When we say that the dynamical system $\Sigma$ is observed, this does not mean that $\Sigma$ is necessarily observable with respect to the output $h$. The problem of constructing flat inputs consists of finding independent control vector fields $g_{1}, \ldots, g_{m}$ (whose inputs $u_{1}, \ldots, u_{m}$ are called flat inputs) such that the control-affine system $\Sigma_{c}$, associated to $\Sigma$, and given by

$$
\Sigma_{c}: \dot{x}=f(x)+\sum_{i=1}^{m} g_{i}(x) u_{i},
$$

is $x$-flat with respect to the the original output $\left(h_{1}, \ldots, h_{m}\right)$. In that case, we will say that the pair $\left(\Sigma_{c}, h\right)$ is $x$-flat. As we have already noticed, flatness, and therefore the problem of constructing flat inputs, is closely related to observability. We denote by $\mathcal{H}(x)$ the observability codistribution (see, e.g., [13])

$$
\mathcal{H}(x)=\operatorname{span}\left\{\mathrm{d} L_{f}^{j-1} h_{i}(x), j \geq 1,1 \leq i \leq m\right\}
$$

associated with the output $h$. Clearly, observability rank condition [13] simply means $\operatorname{dim} \mathcal{H}(x)=n$. Similarly to [20], we will distinguish the observable case $\operatorname{dim} \mathcal{H}(x)=n$ and the unobservable case $\operatorname{dim} \mathcal{H}(x)=k<n$. In [19, 20, we defined the notions of observability quasi-indices for the observable case (see also [33, 14, 3] for related concepts) and respectively, of partial observability quasi-indices for the unobservable one. We briefly recall and discuss them below.

Definition 2.2 (OQI's and POQI's definition [20]). Consider the observed system $(\Sigma, h)$ and let $x_{0} \in \mathbb{X}$. If the system $(\Sigma, h)$ satisfies, in a neighborhood of $x_{0}$,

$$
\begin{gathered}
\operatorname{dim} \mathcal{H}(x)=\text { const. }=k \text { and } \\
\operatorname{dim} \operatorname{span}\left\{\mathrm{d} L_{f}^{j-1} h_{i}(x), 1 \leq j \leq \rho_{i}, 1 \leq i \leq m\right\}=\sum_{i=1}^{m} \rho_{i}=k,
\end{gathered}
$$

then $(\Sigma, h)$ is said to have partial observability quasi-indices $\left(\rho_{1}, \ldots, \rho_{m}\right)$, shortly POQI's, at $x_{0}$ if $k<n$, and observability quasi-indices, shortly OQI's, if $k=n$. Since POQI's and OQI's may depend on a point, we say that quasi-indices $\left(\rho_{1}, \ldots, \rho_{m}\right)$ are uniform in an open subset $X$ of $\mathbb{X}$ if $\left(\rho_{1}, \ldots, \rho_{m}\right)$ form quasi-indices at any $x_{0} \in X$.

Whenever we refer to observability or (local observability), we will mean that the observability rank condition $\operatorname{dim} \mathcal{H}(x)=n$ holds around $x_{0}$ implying that we can distinguish any two points of a neighborhood of $x_{0}$ (which is called strong local observability in [23] because it is stronger than the classical notion of local weak observability of [13] that requires to distinguish $x_{0}$ from its neighbors). By the unobservable case, we mean that $\operatorname{dim} \mathcal{H}(x)<n$. All results hold for systems that possess OQI's (the observable case) or POQI's (the unobservable case).

The existence of OQI's at $x_{0}$ implies local observability of $(\Sigma, h)$. Conversely, if $(\Sigma, h)$ satisfies $\operatorname{dim} \mathcal{H}(x)=n$ for any $x$ in $\mathbb{X}$, i.e., is locally observable everywhere on $\mathbb{X}$, then it possesses OQI's on an open and dense subset of $\mathbb{X}$, see Lemma 2.1 below. The definition of POQI's is more restrictive than just the lack of observability at a point: we require the system to be nowhere observable on a whole neighborhood of $x_{0}$ and, moreover, its observability defect has to be constant on that neighborhood (so only $k$ directions, with $k<n$ constant, can be observed around any point of that neighborhood). Similarly to OQI's, it turns out that on an open and dense subset of $\mathbb{X}$, the lack of observability and the existence of POQI's coincide. Notice that if OQI's or POQI's exist at $x_{0}$ then, in general, they are not unique. From now on, we will denote by calligraphic $\mathcal{X}$ (sometimes with suitable indices) open and dense subsets of $\mathbb{X}$ and by $X$ just open subsets of $\mathbb{X}$. 
Lemma 2.1 (Genericity of constant rank [20]). For any observed dynamics $(\Sigma, h)$ there exists an open and dense subset $\mathcal{X} \subset \mathbb{X}$ on which $\operatorname{dim} \mathcal{H}(x)$ is locally constant and on each connected component $X_{c}$ of $\mathcal{X}$, where $\operatorname{dim} \mathcal{H}(x)=$ const. $=k$ (the value of $k$ may depend on the component), POQI's exist at any $x_{0} \in X_{c}$, if $k<n$, or OQI's exist at any $x_{0} \in X_{c}$, if $k=n$.

In the analytic case, the dimension $k$ is the same on all components $X_{c}$ but it may vary from one $X_{c}$ to another in the $\mathcal{C}^{\infty}$-case (this does not affect our constructions below, that are local, so given on an arbitrary but fixed connected component). We send the reader to [20] for the proof, other discussions, and examples illustrating Lemma 2.1.

Assumption and notation for $\mathcal{X}$. Throughout the paper, we suppose that $(\Sigma, h)$ is either locally observable everywhere on $\mathbb{X}$ (i.e., $\operatorname{dim} \mathcal{H}(x)=n$ for any $x \in \mathbb{X}$ implying that OQI's $\left(\rho_{1}, \ldots, \rho_{m}\right)$ exist on an open and dense subset $\left.\mathcal{X} \in \mathbb{X}\right)$ or nowhere observable (i.e., $\operatorname{dim} \mathcal{H}(x)=k<n$ for any $x \in \mathbb{X}$ implying that POQI's $\left(\rho_{1}, \ldots, \rho_{m}\right)$ exist on an open and dense subset $\mathcal{X} \in \mathbb{X}$ ).

For both cases, observable and non observable, locally around $x_{0} \in \mathcal{X}$, introduce coordinates

$$
w_{i}^{j}=L_{f}^{j-1} h_{i}, \quad 1 \leq j \leq \rho_{i}, 1 \leq i \leq m,
$$

in which the system $\Sigma$ can be locally transformed into

$$
\begin{array}{rlrl}
\dot{w}_{i}^{j} & =w_{i}^{j+1}, & & 1 \leq j \leq \rho_{i}-1, \\
\dot{w}_{i}^{\rho_{i}} & =a_{i}(w), & 1 \leq i \leq m, \\
\dot{z} & =b(w, z), &
\end{array}
$$

where $\left(h_{1}, \ldots, h_{m}\right)=\left(w_{1}^{1}, \ldots, w_{m}^{1}\right)$ and either

(i) $\operatorname{dim} w=n$ and the $z$-subsystem is absent so we are in the observable case (i.e., $\Sigma$ or is locally transformed into (50)

(ii) $\operatorname{dim} w=k<n$ and $\operatorname{dim} w+\operatorname{dim} z=n$ so we are in the unobservable case, where $z$ consists of any coordinates completing $w$ to a coordinate system (for instance, $z$ can be taken as well chosen original coordinates $\left.x_{i_{1}}, \ldots, x_{i_{n-k}}\right)$.

In the unobservable case, representation (5)-(6) will be called observed-unobserved form. The $w$-coordinates are the states observed with the help of the output $h$ and its successive derivatives, and there are $k$ of them. The $z$-coordinates correspond to the unobserved directions, there are $n-k$ (which is the observability defect) of them and they may be affected by $w$, see Figure 1 .

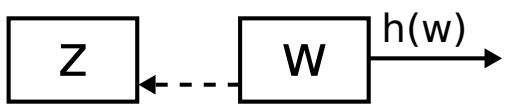

Figure 1: Observed-unobserved variables. 


\section{Main results}

When constructing flat inputs, we modify the original dynamics $\dot{x}=f(x)$ of the system (by adding to $f$ the sum $\sum_{i=1}^{m} u_{i} g_{i}$ ) and therefore we change some of its properties. For some applications, it may be important to modify the original system in a minimal way, namely when some of its properties have to be preserved for the associated flat control system. The issue that we address in this paper is the minimal modification of the original dynamics.

From now on, we work around points of the open and dense subset $\mathcal{X}$ of Lemma 2.1 (on which $(\Sigma, h)$ admits either OQI's or POQI's) and when we refer to $w$-coordinates, we mean the $w$-variables locally defined by (4), with $\left(\rho_{1}, \ldots, \rho_{m}\right)$ being either OQI's or POQI's.

Definition 3.1 (Measure of modification). Consider the observed system $(\Sigma, h)$ in $(w, z)$-coordinates, where $z$ consists of variables completing the $w_{i}^{j}$ s s to a coordinate system (respectively, are absent in the observable case). The measure of modification of $(\Sigma, h)$ by adding flat inputs is the number of nonzero components in the $(w, z)$ coordinates (respectively, in the $w$-coordinates in the observable case) of the control vector fields $g_{1}, \ldots, g_{m}$ that render $\left(\Sigma_{c}, h\right)$ flat.

Equivalently, the measure of modification is the number of equations of (5)-(6) (where subsystem (6) is absent in the observable case), that we have to change by adding the flat-inputs $u_{1}, \ldots, u_{m}$ (each modified equation is supposed to be affected by one flat input only, which is the case for all constructions of [20]).

The control vector fields $g_{1}, \ldots, g_{m}$ have together at least $m$ nonzero components (at least one for each $g_{i}$ ), so at least $m$ equations of the original dynamics (5)-(6) have to be modified to achieve flatness. We will see that we can always construct control vector fields that distort $m$ equations only (which is the minimal possible) if $(\Sigma, h)$ is observable (Theorem 3.1), while in the unobservable case, the best that we can hope for is to modify $m+1$ equations only to involve controls (Theorem 3.2). All results (except for those whose proofs are immediate) are proved in Section 5

Theorem 3.1. Consider the observed system $(\Sigma, h)$ around $x_{0} \in \mathcal{X}$. There locally exist control vector fields $g_{1}, \ldots, g_{m}$ that have together, in the $(w, z)$-coordinates, with $w$ given by (4), the minimal possible, which is $m$, nonzero components and render $\left(\Sigma_{c}, h\right)$ flat at $x_{0}$ if and only if $(\Sigma, h)$ is locally observable at $x_{0}$ (and thus $z$-coordinates are, actually, absent and $\Sigma$ can be locally brought into (5)).

Proof. The proof, based on Remark 3.2 below, is given in Section 5.1

Remark 3.1 (Single-output case). In the single-output case (i.e., $m=1$ and $y=h(x) \in$ $\mathbb{R})$, a flat input can be constructed if and only if the system $\Sigma$ together with its output $h$ is locally observable [32]. Thus if $(\Sigma, h)$ can be rendered flat, then in the $w$-coordinates, the vector field $g_{1}$ (there is only one since $m=1$ ) leading to a flat $\left(\Sigma_{c}, h\right)$ can always be chosen with one nonzero component only.

The above theorem recalls very much Theorem 2.1 of [20] stating that the observed system $(\Sigma, h)$ can be made flat of differential weight $n+m$ (which is the minimal possible differential weight of a flat system with $n$ states and $m$ controls) if and only if it admits OQI's.

Remark 3.2 (Minimally modified normal form and minimal differential weight). If $(\Sigma, h)$ admits OQI's $\left(\rho_{1}, \ldots, \rho_{m}\right)$ at $x_{0}$, then $\Sigma$ can be locally brought into (5) (the 
$z$-subsystem (6) being absent in the observable case). For form (5), we define the control vector fields $g_{i}(w)=\frac{\partial}{\partial w_{i}^{\rho_{i}}}, 1 \leq i \leq m$, that have together the minimal possible, which is $m$, nonzero components and for which the corresponding control system is given by

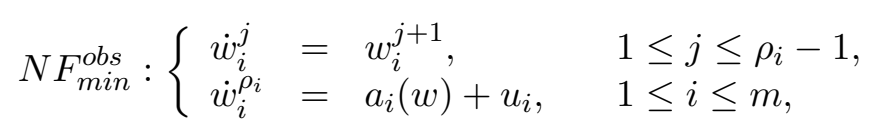

with $h=\left(w_{1}^{1}, \ldots, w_{m}^{1}\right)$ a flat output of differential weight $n+m$, the minimal possible. Notice that the minimal modification of $\Sigma$ is compatible with flatness of minimal differential weight. So in the observable case, we can always render the system $(\Sigma, h)$ flat of minimal differential weight $n+m$ by modifying $m$ equations only of the original system (5) when adding flat inputs. Moreover, the associated control vector fields can be computed with the following system of algebraic equations.

Corollary 3.1. In a local coordinate system $x=\left(x_{1}, \ldots, x_{n}\right)$, form the $n \times n$-matrix $H(x)$ whose first $n-m$ rows are the differentials $\mathrm{d} L_{f}^{j-1} h_{i}(x), 1 \leq j \leq \rho_{i}-1$, and the last $m$ rows are the differentials $\mathrm{d} L_{f}^{\rho_{i}-1} h_{i}(x), 1 \leq i \leq m$, where $\left(\rho_{1}, \ldots, \rho_{m}\right)$ are OQI's at $x_{0}$. Then around $x_{0}$ the matrix $H(x)$ is invertible, and the control vector fields leading to a minimally modified flat control system can be computed by

$$
g(x)=(H(x))^{-1} \cdot\left(\begin{array}{c}
0 \\
I_{m}
\end{array}\right)
$$

where $g(x)=\left(g_{1}(x) \ldots g_{m}(x)\right), 0=0_{(n-m) \times m}$ and $I_{m}$ is the identity $m \times m$-matrix.

Proof. Follows directly from Theorem 3.1 and Remark 3.2 .

Finally, observe that in the case characterized by Theorem 3.1 and leading to the flat control system $\left(\mathrm{NF}_{\mathrm{min}}^{\mathrm{obs}}, h\right)$, even if the construction of the control vector fields $g_{1}, \ldots, g_{m}$ is local around a given $x_{0}$, description (2) for $\left(\mathrm{NF}_{\min }^{\mathrm{obs}}, h\right)$ is always global with respect to the control $u$, so we never face flatness singularities in the control space.

The following result is a direct consequence of Theorem 3.1 .

Corollary 3.2. Suppose that $(\Sigma, h)$ is not locally observable at $x_{0}$ and that it admits POQI's at $x_{0}$. If $(\Sigma, h)$ has flat inputs at $x_{0}$, then any choice of control vector fields $g_{1}, \ldots, g_{m}$ leading to a flat control system $\left(\Sigma_{c}, h\right)$ have together, in $(w, z)$-coordinates, with $z$ consisting of any variables completing $w=\left(w_{i}^{j}\right)$ to a coordinate system, at least $m+1$ nonzero components.

Proof. Follows directly from Theorem 3.1 .

Remark 3.3. According to the above corollary, in the unobservable case, the best we can hope for is that only $m+1$ equations involve the control. However, in many cases, we can obtain from $\left(\Sigma_{c}, h\right)$ by rectifying the control vector fields $g_{1}, \ldots, g_{m}$ (if possible, i.e., if the distribution spanned by them is involutive) a feedback equivalent flat control system $\left(\widetilde{\Sigma}_{c}, h\right)$ with $m$ modified equations only, see Corollary 3.5 in Section 3.3.2. Notice, however, that in order to get $\left(\widetilde{\Sigma}_{c}, h\right)$, the distribution $\mathcal{G}=$ span $\left\{g_{1}, \ldots, g_{m}\right\}$, which is not given a priori, must be known. So first, we have to compute in an optimal way $g_{1}, \ldots, g_{m}$ via our constructions (explained in Theorems 3.2 and 3.3 below) and only then, to introduce new coordinates, necessarily mixing the observed $w$-variables and the unobserved $z$-variables, in which the control vector fields are rectified. 


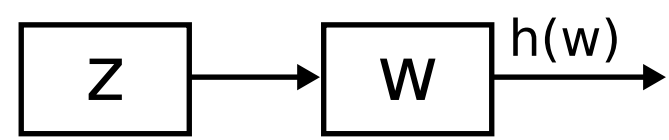

(a) Link needed for observability.

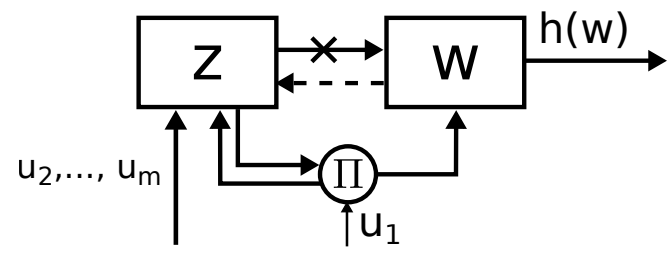

(c) Observability obtained with the $g_{i}$ 's defined in [20].

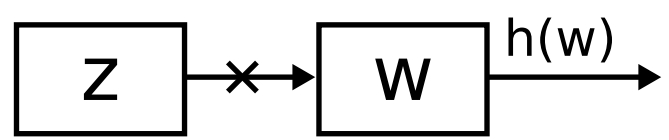

(b) No link between the $w$ - and the $z$-subsystems.

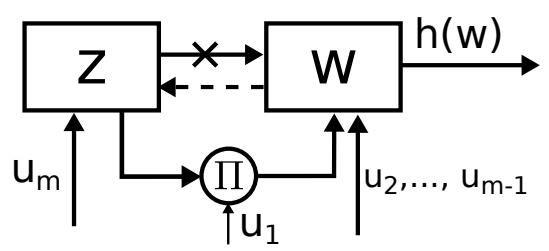

(d) Observability obtained with the $g_{i}$ 's defined by Theorem 3.2

Figure 2: Rendering the system observable.

\subsection{Constructing flat inputs for a minimal modification of $\Sigma$}

From now on, we assume that $m \geq 2$ and that $(\Sigma, h)$ is nowhere observable, so by Lemma 2.1. POQI's $\left(\rho_{1}, \ldots, \rho_{m}\right)$, with $\rho_{1}+\ldots+\rho_{m}=k<n$, exist on an open and dense subset $\mathcal{X} \in \mathbb{X}$, on which we work. Two questions arise:

Question 3.1. How can the original $(\Sigma, h)$ be minimally modified in $(w, z)$-variables (i.e., $m+1$ modified equations) by adding flat inputs around $x_{0}$ ?

Question 3.2. Can we always construct flat inputs minimally modifying $(\Sigma, h)$ at $x_{0}$ ?

Theorem 3.2 (which, together with Theorem 3.3, is the main result of the paper) answers these questions and gives conditions allowing the construction of vector fields $g_{1}, \ldots, g_{m}$ modifying $m+1$ equations only, which is the minimal possible in the unobservable case (instead of $m$ in the observable case). A comparison between Theorem 3.2 and the results of [20] is discussed in Section 3.2. Theorem 3.2 uses a normal form, denoted $\mathrm{NF}_{\text {min }}$, and the construction of $\mathrm{NF}_{\text {min }}$ is based, as that of the forms of [20], on the following idea: a flat system is observable (with respect to its flat output and independently of the applied input signal), so we have to render the original system $(\Sigma, h)$ observable. For observability we need a link from the $z$-subsystem towards the observed $w$-subsystem, but for $\Sigma$ there is no such a link, see the observed-unobserved form (5)-(6). It follows that we have to create a link assuring observability with the help of the control vector fields, see Figure 2, where $\Pi$ stands for products of some $z$-variables and $u_{1}$. Notice also that it is crucial to distinguish the observability (or unobservability) of controlled systems from observability of uncontrolled ones (recall that for nonlinear systems, the observability property depends on the control [9, 11]). Here we deal with unobservable uncontrolled systems that become (at least) locally observable due to a suitable design of flat inputs.

For $1 \leq i \leq m$, the variables $w_{i}^{j}, 1 \leq j \leq \rho_{i}$, define the $w_{i}$-chain. The $z$-variables of $\mathrm{NF}_{\text {min }}$ are indexed $z=\left(z^{1}, \ldots, z^{n-k}\right)$. The following notations will be used in condition (M3) of Theorem 3.2. Suppose that $x_{0} \in \mathcal{X}$ is an equilibrium, that is, $f\left(x_{0}\right)=0$, and consider the $w$-coordinates, given by (4) and completed by $z$ to a local coordinate system $(w, z)$. Represent the system in $(w, z)$-coordinates $(\dot{w}, \dot{z})=(a(w), b(w, z))$ and define the matrix

$$
A=\frac{\partial b}{\partial z}\left(w_{0}, z_{0}\right)
$$


Theorem 3.2. Consider the observed system $(\Sigma, h)$ around any $x_{0} \in \mathcal{X}$, that is, $(\Sigma, h)$ admits POQI's $\left(\rho_{1}, \ldots, \rho_{m}\right)$ at $x_{0}$.

(M1) Suppose that there exists a smooth function $\psi: \mathcal{O}_{x_{0}} \mapsto \mathbb{R}$, where $\mathcal{O}_{x_{0}}$ is a neighborhood of $x_{0}$, completing $h$ to a new output $h^{1}=\left(h_{1}, \ldots, h_{m}, \psi\right)$ such that $\left(\Sigma, h^{1}\right)$ is locally observable at $x_{0}$ and admits OQI's $\left(\rho_{1}, \ldots, \rho_{m}, n-k\right)$ at $x_{0}$. Then there exist local coordinates $(w, z)$, with $w$ given by (4) and $z$ consisting of

$$
z^{q}=L_{f}^{q-1} \psi(x), 1 \leq q \leq n-k,
$$

that bring $(\Sigma, h)$ into (5)-(6) with the unobserved subsystem (6) given by:

$$
\begin{cases}\dot{z}^{q} & =z^{q+1}, \quad 1 \leq q \leq n-k-1 \\ \dot{z}^{n-k} & =b^{n-k}(w, z)\end{cases}
$$

where $b^{n-k}$ is a smooth function. For this form, we can always locally construct $g_{1}, \ldots, g_{m}$ that have together the minimal possible, which is $m+1$, number of nonzero components, and such that $\left(\Sigma_{c}, h\right)$ is $x$-flat at $x_{0}$, and in $(w, z)$-coordinates is given by the following form:

$$
N F_{\text {min }}\left\{\begin{aligned}
\dot{w}_{i}^{j}=w_{i}^{j+1}, & \dot{w}_{m}^{j}=w_{m}^{j+1} \\
\dot{w}_{i}^{\rho_{i}}=a_{i}(w)+u_{i}, & \dot{w}_{m}^{\rho_{m}}=a_{m}(w)+z^{1} u_{1}, \\
1 \leq i \leq m-1, & \dot{z}^{q}=z^{q+1} \\
& \dot{z}^{n-k}=b^{n-k}(w, z)+u_{m}
\end{aligned}\right.
$$

where $1 \leq j \leq \rho_{i}-1,1 \leq q \leq n-k-1$, and $h=\left(w_{1}^{1}, \ldots, w_{m}^{1}\right)$ is a flat output of differential weight at least $n+m+(n-k)$.

(M2) If $f\left(x_{0}\right) \neq 0$, then a smooth function $\psi$ satisfying the assumption of condition (M1) always exists and, therefore, we can always locally construct the minimally modified flat control system $N F_{\min }$.

(M3) If $f\left(x_{0}\right)=0$, then the existence of a function $\psi$ satisfying condition (M1) is equivalent to the existence of a row vector $C \in \mathbb{R}^{1 \times(n-k)}$ such that

$$
\operatorname{rk}\left(\begin{array}{c}
C \\
C A \\
\vdots \\
C A^{n-k-1}
\end{array}\right)=n-k
$$

in which case a solution of (M1) is $\psi=C z$.

Proof. The proof, based on the following algorithm, is given in Section 5.2 .

Remark 3.4 (Singularities in the control space). We would like to emphasize that singularities in the control space are unavoidable (recall that we defined flatness at $x_{0}$ as a generic property with respect to the control $u$, see Definition 2.1). Indeed, if the system $(\Sigma, h)$ is not observable, we add to $f$ the sum $\sum_{i=1}^{m} u_{i} g_{i}$ to render it observable. Now it is clear that if the components of $u$, that form a link between the originally observable and unobservable subsystems, are zero, then the link is broken, the system $\left(\Sigma_{c}, h\right)$ looses its observability and, as a consequence, is not flat for those control values. In the above form only the vector field $g_{1}$ (and thus only the input $u_{1}$ ) is used for the link between the observed $w$-subsystem and the unobserved $z$-subsystem, see Figure $2 \mathrm{~d}$, while the remaining $u_{2}, \ldots, u_{m}$ affect the observed $w$-subsystem only. Hence it is clear that any control $u=\left(u_{1}, u_{2}, \ldots, u_{m}\right)$, for which $u_{1}=0$, creates singularities of observability (and thus of flatness). 


\subsubsection{Algorithm for constructing $g_{1}, \ldots, g_{m}$ of the normal form $\mathbf{N F}_{\text {min }}$}

Consider the system $(\Sigma, h)$ around any $x_{0} \in \mathcal{X}$, so $(\Sigma, h)$ admits POQI's $\left(\rho_{1}, \ldots, \rho_{m}\right)$ at $x_{0}$. Suppose that there exists a smooth function $\psi$ satisfying the assumption of (M1). It follows that $\mathrm{d} L_{f}^{q-1} \psi(x)$, for $1 \leq q \leq n-k$, are independent at $x_{0}$ modulo $\mathcal{H}\left(x_{0}\right)$, thus $(w, z)$, with $w$ and $z$ given, respectively, by (4) and (9), is a valid change of coordinates around $x_{0}$ bringing the system $(\Sigma, h)$ into the observed-unobserved form (5)-(6) with the unobserved subsystem being of form (10). For that form define the control vector fields:

$$
g_{1}=\frac{\partial}{\partial w_{1}^{\rho_{1}}}+z^{1} \frac{\partial}{\partial w_{m}^{\rho_{m}}}, \quad g_{i}=\frac{\partial}{\partial w_{i}^{\rho_{i}}}, \text { for } 2 \leq i \leq m-1, \quad \text { and } \quad g_{m}=\frac{\partial}{\partial z^{n-k}},
$$

that have together $m+1$ nonzero components. The associated control system is in the form $\mathrm{NF}_{\text {min }}$ which is clearly $x$-flat at $x_{0}$ with $h=\left(w_{1}^{1}, \ldots, w_{m}^{1}\right)$ being a flat output of differential weight at least $n+m+(n-k)$ since $u_{1}$ is differentiated $(n-k)$-times and, perhaps, some other controls as well (see the last paragraph of Section 3.1 .2 where this is discussed in details). $\triangleleft$

The above algorithm provides an explicit construction, that uses differentiation and algebraic operations only, of $g_{i}$ 's, given by the following proposition.

Proposition 3.1. Consider the observed dynamical system $(\Sigma, h)$ around any $x_{0} \in \mathcal{X}$ and suppose that a function $\psi$ satisfying the assumption of Theorem 3.2 (M1) exists. Denote $\psi^{q}=L_{f}^{q-1} \psi$, for $1 \leq q \leq n-k$, and $\varphi_{i}^{j}=L_{f}^{j-1} h_{i}$, for $1 \leq j \leq \rho_{i}, 1 \leq i \leq m$. Vector fields $g_{1}, \ldots, g_{m}$ such that the $x$-flat control system $\left(\Sigma_{c}, h\right)$ is minimally modified can be constructed by

$$
\begin{aligned}
& <\mathrm{d} \varphi_{i}^{j}, g_{l}>=0,1 \leq i, l \leq m, 1 \leq j \leq \rho_{i}-1, \\
& <\mathrm{d} \varphi_{i}^{\rho_{i}}, g_{l}>=\left\{\begin{array}{l}
\delta_{i l}, 1 \leq i, l \leq m,(i, l) \neq(m, 1),(m, m), \\
\psi^{1},(i, l)=(m, 1), \\
0, \quad(i, l)=(m, m),
\end{array}\right.
\end{aligned}
$$

and

$$
<\mathrm{d} \psi^{q}, g_{l}>=\left\{\begin{array}{l}
0,1 \leq l \leq m, 1 \leq q \leq n-k,(q, l) \neq(n-k, m) \\
1,(q, l)=(n-k, m),
\end{array}\right.
$$

where $\delta_{i l}=1$, if $i=l$, and 0 , otherwise.

Corollary 3.3. In a local coordinate system $x=\left(x_{1}, \ldots, x_{n}\right)$, form the $n \times n$-matrix $H^{1}(x)=\left(\begin{array}{c}H(x) \\ \Psi(x)\end{array}\right)$ whose first $k$ rows $H(x)$ are the differentials $\mathrm{d} \varphi_{i}^{j}(x)=\mathrm{d} L_{f}^{j-1} h_{i}(x)$ and the last $n-k$ rows $\Psi(x)$ are the differentials $\mathrm{d} \psi^{q}=\mathrm{d} L_{f}^{q-1} \psi$, where we use the notations of Proposition 3.1. Then equations (13)-(15) are equivalent to

$$
H^{1}(x) \cdot g(x)=D(x)
$$

where $g(x)=\left(g_{1}(x) \ldots g_{m}(x)\right)$, and $D(x)=\left(d_{i}^{s}(x)\right)$, with $d_{i}^{s}$, for $1 \leq s \leq n, 1 \leq i \leq m$, denoting suitable terms of the right hand-side of (13)-(15). If the function $\psi$ satisfies the assumption of Theorem 3.2 (M1), then the matrix $H^{1}(x)$ is invertible around $x_{0}$ and

$$
g(x)=\left(H^{1}(x)\right)^{-1} \cdot D(x) .
$$

Proof. The proofs of Proposition 3.1 and Corollary 3.3 follow directly from the algorithm in Section 3.1 .1 for constructing $g_{1}, \ldots, g_{m}$ of $\mathrm{NF}_{\text {min }}$. 


\subsubsection{Discussion of $\mathrm{NF}_{\min }$}

In the unobservable case, as asserted by Corollary 3.2, the minimal possible number of nonzero components of $g_{1}, \ldots, g_{m}$ yielding a flat control system $\left(\Sigma_{c}, h\right)$ is $m+1$. Indeed, we necessarily have to invent at least one nonzero component per $w_{i}$-chain involving the inputs: we cannot achieve flatness by modifying less that $m$ equations of the $w$-part and those equations can involve at most $m-1$ different flat inputs (because involving all $m$ controls would contradict flatness, since $\operatorname{dim} w=k<n)$. The remaining inputs necessarily affect the $z$-part (there are at least as many modified $z$-equations as the number of inputs not involved in the $w$-subsystem). So, since the goal is to minimally modify the original dynamics (i.e., the control vector fields should have exactly $m+1$ nonzero components in $(w, z)$-coordinates), the $w$-subsystem of a minimally modified flat control system $\left(\Sigma_{c}, h\right)$ necessarily contains $m$ modified equations and has to involve the maximal number of flat inputs, that is, $m-1$, implying that only one equation of the $z$ part should be modified by adding a control. Moreover, a link between the $w$-subsystem and the $z$-subsystem has to be created and for a minimally modified flat control system only one $w$-component of one control vector field among $g_{1}, \ldots, g_{m}$ is needed for that link, that is, has to depend on $z$ (that component will be called a linking term). In our construction the linking term is defined by the function $\psi$ satisfying the condition (M1) of Theorem 3.2. By (M1), $n-k$ successive derivatives of $\psi$ along the drift $f$ are independent modulo $\mathcal{H}$, thus adding $\psi u_{1}=z^{1} u_{1}$ to $\dot{w}_{m}^{\rho_{m}}=a_{m}$ and $u_{i}$ to $\dot{w}_{i}^{\rho_{i}}=a_{i}$, $1 \leq i \leq m-1$, (actually, instead we can add $z^{1} u_{1}$ to any equation $\dot{w}_{l}^{\rho_{l}}=a_{l}$, for a certain $1 \leq l \leq m$, and $u_{i}$ to the remaining $\left.\dot{w}_{i}^{\rho_{i}}=a_{i}, i \neq l\right)$, render the overall system $\Sigma_{c}$ observable. The only control affecting the $z$-structure should appear at the bottom level (should affect $\dot{z}^{n-k}=\frac{\mathrm{d}}{\mathrm{d} t}\left(L_{f}^{n-k-1} \psi\right)$ only). Notice that adding $\psi u_{1}=z^{1} u_{1}$ to $\dot{w}_{m}^{\rho_{m}}=a_{m}$ (and $u_{i}$ to $\dot{w}_{i}^{\rho_{i}}=a_{i}$ ) renders the control system $\Sigma_{c}$ observable if and only if $\psi$ completes the original output $h$ to a new output $h^{1}=(h, \psi)$ that renders the original uncontrolled system $\left(\Sigma, h^{1}\right)$ locally observable around the nominal point $x_{0}$, which explains the role of condition (M1). If we work around a nonequilibrium point $x_{0}$ (i.e., $f\left(x_{0}\right) \neq 0$ ), such a function $\psi$ always exists and can be explicitly computed (see the proof of (M2) in Section 5.2), and thus we can always locally construct a minimally modified flat control system $\left(\mathrm{NF}_{\min }, h\right)$. Necessary and sufficient conditions for the case of an equilibrium $f\left(x_{0}\right)=0$ are given by condition (M3) and in this case, the problem of computing $\psi$ reduces, actually, to a linear one.

It is clear that $h=\left(w_{1}^{1}, \ldots, w_{m}^{1}\right)$ is a flat output of the form $\mathrm{NF}_{\min }$ and its differential weight is at least $n+m+(n-k)$. Indeed, we have $w_{i}^{j}=h_{i}^{(j-1)}$, for $1 \leq i \leq m$, $1 \leq j \leq \rho_{i}$, and $h_{i}^{\left(\rho_{i}\right)}=\dot{w}_{i}^{\rho_{i}}=a_{i}(w)+u_{i}, 1 \leq i \leq m-1$, from which we express $u_{i}$, for $1 \leq i \leq m-1$. Then, provided that $u_{10} \neq 0$, the equation $h_{m}^{\left(\rho_{m}\right)}=\dot{w}_{m}^{\rho_{m}}=a_{m}(w)+z^{1} u_{1}$ allows to express $z^{1}$. By differentiating $z^{1}$, we get $z^{2}$, and so on. It follows that $u_{1}$ necessarily has to be differentiated $n-k$ times in order to express all remaining $z$-states and control $u_{m}$ with the help the $h_{i}$ 's and their derivatives, and in the best case, it is the the only input among $u_{1}, \ldots, u_{m-1}$ that needs to be differentiated. Notice however that, in general, the function $a_{m}(w)$ depends on all $w$-coordinates, so when differentiating it, other inputs among $u_{2}, \ldots, u_{m-1}$ and their derivatives may appear (thus increasing the differential weight of $h$ ).

\subsection{Comparison with the normal forms of [20]}

This section is devoted to discussing $\mathrm{NF}_{\text {min }}$ in comparison with the results of [20] (which are also based on normal forms). In addition to the number of equations modified by adding flat inputs, we focus namely on three aspects: the construction of the forms and, 
in particular, the choice of the linking terms (i.e., the nonzero components of $g_{1}$ ), the minimal differential weight of $h$ as a flat output of each form and, finally, the number of flat inputs affecting the $z$-subsystem for each form.

We start by briefly presenting the three normal forms NF1, NF2 and NF3 of [20] (we send the reader to the aforementioned paper for algorithms of construction, proofs and detailed discussions). We distinguish two cases: $n-k \geq m-1$ (corresponding to NF1 and NF2) and $n-k<m-1$ (corresponding to NF3).

Normal form NF1 $(\operatorname{dim} z=n-k \geq m-1)$. The construction of NF1 is performed on an open and dense subset of $\mathbb{X}$ and is valid around any point $x_{0}$ of that set (equilibrium or not). The idea is to complete the original output $h$ to a new output $h^{\ell}=\left(h, \psi^{1}, \ldots, \psi^{\ell}\right)$ such that $\left(\Sigma, h^{\ell}\right)$ is locally observable, where each $\psi^{s}, 1 \leq s \leq \ell-1$, denotes an $(m-1)$-tuple of functions $\left(\psi_{2}^{s}, \ldots, \psi_{m}^{s}\right)$ (the last one $\psi^{\ell}$ contains $m^{\prime}-1$ functions, with $m^{\prime} \leq m$ ) that always exist and can be arbitrarily chosen (provided that they respect a nonregularity condition). The integers $\left(\rho, \nu^{1}, \ldots, \nu^{\ell}\right)$, where $\rho=\left(\rho_{1}, \ldots, \rho_{m}\right)$ and $\nu^{s}=\left(\nu_{2}^{s}, \ldots, \nu_{m}^{s}\right)$, for $1 \leq s \leq \ell$ (where $\bar{m}$ depends on $s$ and equals either $m$ or $m^{\prime}$, see equation (18) below), of NF1 are OQI's of $\left(\Sigma, h^{\ell}\right)$. The variables $z_{i}^{s, q}$ of NF1 are suitable successive Lie derivatives of $\psi_{i}^{s}$ with respect to $f$. They form $\ell$ blocks, for $s=1, \ldots, \ell$. In each block, the index $q$ corresponds to successive derivatives and the index $s$ to subsystems. There are $\ell$ subsystems, each of them containing $m-1$ chains $z_{i}^{s}$ (indexed by $i$, for $2 \leq i \leq m$ ), with the exception of the $\ell$-th subsystem, where there are $m^{\prime}-1$ chains $z_{i}^{s}$ (indexed by $i$, for $2 \leq i \leq m^{\prime}$ ). This justifies the following notation:

$$
\bar{m}=\left\{\begin{array}{ll}
m, \text { for } 1 \leq s \leq \ell-1, \\
m^{\prime}, \text { for } s=\ell,
\end{array} \text { and } \bar{\ell}= \begin{cases}\ell, & \text { for } 2 \leq i \leq m^{\prime}, \\
\ell-1, & \text { for } m^{\prime}+1 \leq i \leq m .\end{cases}\right.
$$

Finally, for $1 \leq s \leq \ell$, denote $\bar{z}^{s}=\left(z_{i}^{j, q}, 1 \leq q \leq \nu_{i}^{j}, 1 \leq j \leq s, 2 \leq i \leq \bar{m}\right)$, i.e., $\bar{z}^{s}$ consists of all components of first $s$ blocks. The normal form $(\mathrm{NF} 1, h)$ is $x$-flat at $x_{0}$ and is given by:

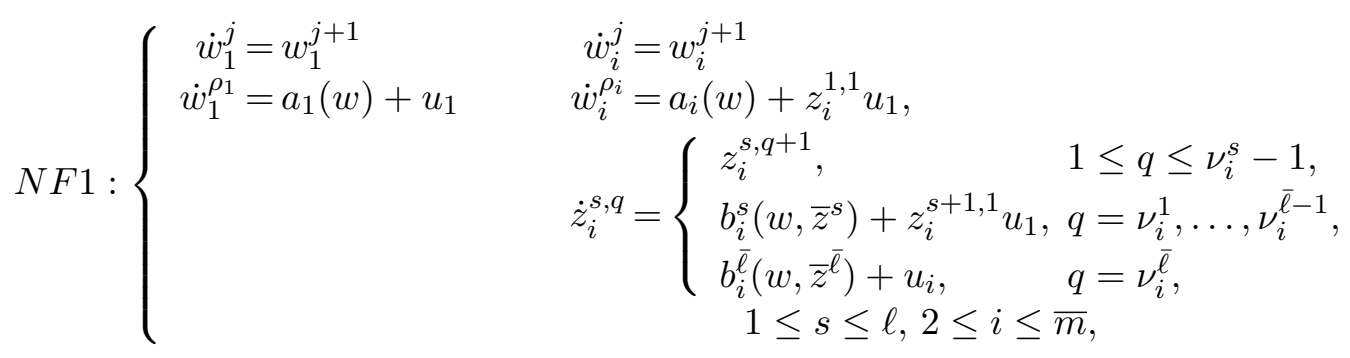

where $1 \leq j \leq \rho_{i}-1$ and $h=\left(w_{1}^{1}, \ldots, w_{m}^{1}\right)$ is a flat output of differential weight at least $n+m+\max _{2 \leq i \leq \bar{m}}\left\{\nu_{i}^{1}+\cdots+\nu_{i}^{\bar{\ell}}\right\}$.

Remark 3.5 (Number of modified equations for NF1). The vector fields $g_{1}, \ldots, g_{m}$ modify together $m+\sum_{i=2}^{m} \bar{\ell}_{i}$ equations (where $\bar{\ell}_{i}$ indicates that $\bar{\ell}$ depends on $i$, see (18)) and since $\bar{\ell} \geq 1$, for all $2 \leq i \leq m$, there are at least $2 m-1$ modified equations. Notice that only $2 m-1$ equations are modified if $\ell=1$ (and only in that case!), that is, only one $(m-1)$-tuple of dummy outputs $\psi^{1}=\left(\psi_{2}^{1}, \ldots, \psi_{m}^{1}\right)$ is need to achieve observability.

Normal form NF2 $(\operatorname{dim} z=n-k \geq m-1)$. Recall that $\operatorname{dim} \mathcal{H}(x)=k$ and let

$$
n-k=p(m-1)+r \quad \text { and } \quad p^{*}=\left\{\begin{array}{l}
p, \text { if } r=0 \\
p+1, \text { if } r>0
\end{array}\right.
$$


be the Euclidean division of $n-k$ (the observability defect) and $m-1$ (the number of output components being $m$ ). Define

$$
\mu_{i}=\left\{\begin{array}{l}
p, \quad 2 \leq i \leq m-r, \\
p+1, m-r+1 \leq i \leq m,
\end{array} \quad \text { and } \quad b_{i}= \begin{cases}1, & i=2 \\
0, & 3 \leq i \leq m\end{cases}\right.
$$

that will correspond, respectively, to the lengths and to the last components of the $z_{i^{-}}$ chains of NF2. The normal form NF2 is valid around any $x_{0} \in \mathbb{X}$ (not just on an open and dense subset as NF1 is) satisfying $f\left(x_{0}\right) \neq 0$ and such that $(\Sigma, h)$ admits POQI's $\left(\rho_{1}, \ldots, \rho_{m}\right)$ at $x_{0}$. The $z$-variables of NF2 are well chosen coordinates in which the drift of the unobserved $z$-part is rectified. $(\mathrm{NF} 2, h)$ is $x$-flat at $x_{0}$ of differential weight $n+m+p^{*}$, and is given by the following form with $h=\left(w_{1}^{1}, \ldots, w_{m}^{1}\right)$ :

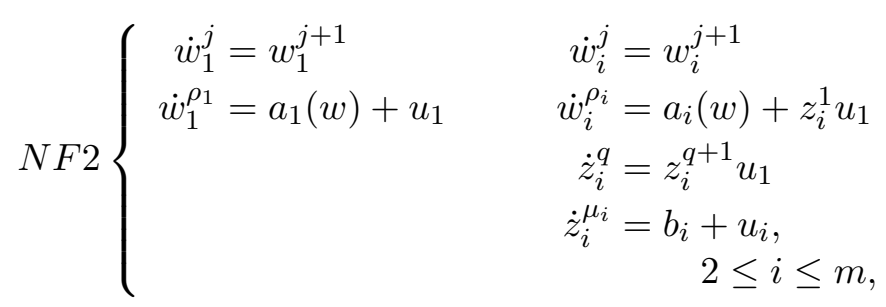

where $1 \leq j \leq \rho_{i}-1,1 \leq q \leq \mu_{i}-1$.

Remark 3.6 (Number of modified equations for NF2). The vector fields $g_{1}, \ldots, g_{m}$ of NF2 modify $m+n-k$ equations of the original dynamics.

Remark 3.7 (Differential weight of $h$ as a flat output of $\Sigma_{c}$ ). In [20], we showed that if there exist $g_{1}, \ldots, g_{m}$ such that $\left(\Sigma_{c}, h\right)$ is locally $x$-flat, then the differential weight of $h$ as a flat output of $\Sigma_{c}$ is at least $n+m+p^{*}$. In particular, the $g_{i}$ 's defining NF2 give the lowest (minimal possible) differential weight of $h$ among all choices of $g_{1}, \ldots, g_{m}$.

Normal form NF3 $(\operatorname{dim} z=r<m-1)$. The normal forms NF1 and NF2 apply when the number of unobserved (with respect to the original output $h$ ) directions is at least $m-1$. A third normal form NF3 is proposed for the case when $\operatorname{dim} z=r<m-1$. NF3 holds around any $x_{0} \in \mathbb{X}$ at which $(\Sigma, h)$ admits POQI's and is given by

$$
N F 3\left\{\begin{array}{rlrl}
\dot{w}_{i}^{j}=w_{i}^{j+1} & \dot{w}_{i}^{j} & =w_{i}^{j+1} \\
\dot{w}_{i}^{\rho_{i}}=a_{i}(w)+u_{i}, & \dot{w}_{i}^{\rho_{i}} & =a_{i}(w)+z_{i}^{1} u_{1}, \\
1 \leq i \leq m^{\prime}, & \dot{z}_{i}^{1}= & b_{i}(w, z)+u_{i}, \\
& m^{\prime}+1 \leq i \leq m,
\end{array}\right.
$$

where $m^{\prime}=m-r, z=\left(z_{m^{\prime}+1}^{1}, \ldots, z_{m}^{1}\right)$ are any coordinates completing the $w$ to a local coordinate system, and $b_{i}(w, z)$ are functions of $w$ and $z$.

Remark 3.8 (Number of modified equations for NF3). The vector fields $g_{1}, \ldots, g_{m}$ of NF3 modify $m+r$ equations of the original dynamics.

\subsubsection{Linking terms and differential weight}

For $\mathrm{NF}_{\min }$ and all forms of [20], the link between the observed $w$-subsystem and the unobserved $z$-subsystem is made with the help of the control vector field $g_{1}$ only and the remaining inputs appear at the bottom level of each $z_{i}$-chain. Contrary to NF1 and NF2 for which the $z$-subsystem is affected by $m-1$ inputs, for $\mathrm{NF}_{\min }$, one control only appears in the $z$-part (recall that, as we have discussed in Section 3.1.2, a minimally modified flat control system should contain only one $z$-chain, which is the case of $\mathrm{NF}_{\min }$ ). The 
construction of the $z$-variables of $\mathrm{NF}_{\min }$ is closely related to the dummy output $\psi$ defining the linking term between the observed $w$-subsystem and the unobserved $z$-subsystem. For NF1 also, the $z$-coordinates are constructed with the help of the dummy outputs $\psi_{i}^{1}, \ldots, \psi_{i}^{\ell}$ defining, respectively, the variables $z_{i}^{1,1}, \ldots, z_{i}^{\ell, 1}$. These functions are the linking terms of NF1, they connect the $w$-subsystem and the first $z$-block as well as the $z$-blocks among them. So for NF1, there are many linking terms and their choice is far from being unique (they are actually arbitrary functions satisfying a nonregularity condition).

The form NF3 is similar to NF1, the main difference between them being that the unobserved $z$-part of NF3 can be affected by at most $r$ inputs, so the remaining controls necessarily appear in the $w$-equations (recalling $\mathrm{NF}_{\text {min }}$ ). NF3 has $r$ linking terms that are, like for NF1, arbitrary functions satisfying a nonregularity condition and defining the $z$-coordinates.

For NF2, the $z$-variables are special. Namely, they are coordinates in which the drift of the unobserved part is rectified (i.e., are given by $n-k-1$ independent, modulo $\mathrm{d} w$, solutions of the first order partial differential equation $L_{f} \gamma(x)=0$ completed by one solution of $L_{f} \gamma(x)=1$ ), so the linking terms between the $w$ - and the $z$-subsystems are not arbitrary either. There is however a significant freedom in choosing the linking terms of NF2. Indeed, once independent solutions of $L_{f} \gamma(x)=0$ have been computed, any $m-1$ of them can play the role of linking terms.

For all forms, the components of the vector fields $g_{1}, \ldots, g_{m}$ depend on the unobserved (with respect to the original output $h$ ) states only and, for NF2 they are designed to be in the multi-chained form. This particular form guarantees that the differential weight of $h$ as a flat output of NF2 is the minimal possible (equal to $n+m+p^{*}$, see Remark 3.7 and relation (19) defining $p^{*}$ ) among all differential weights of $h$ as a flat output of any $\Sigma_{c}$. The differential weight of $h=\left(w_{1}^{1}, \ldots, w_{m}^{1}\right)$ as a flat output of NF3 is also the minimal possible $n+m+1$ (because each $z_{i}$-chain of NF3 is of length one). On the other hand, there is no reason for all $z_{i}$-chains of NF1 to be (contrary to those of NF2 or NF3) of length $p$ or $p+1$, thus the differential weight of $h$ as a flat output of NF1 is, in general, greater than $n+m+p^{*}$.

The differential weight of $h$ as a flat output of the form $\mathrm{NF}_{\min }$ is never (except for the two extreme cases discussed in Section 3.2 .2 below) the minimal possible. Moreover, the differential weight of $h$ as a flat output of the form $\mathrm{NF}_{\min }$ is always (except, again, for the two extreme cases below) greater than that of $h$ as a flat output of NF1 which, in turn, is in general greater than $n+m+p^{*}$.

Example 3.1. Consider the following three-output system which is already in the observed-unobserved form:

$$
\begin{aligned}
\dot{w}_{i}^{j} & =w_{i}^{j+1}, 1 \leq j \leq \rho_{i}-1 \\
\dot{w}_{i}^{\rho_{i}} & =a_{i}(w), 1 \leq i \leq 3 \\
\dot{z}_{1} & =z_{2} \\
\dot{z}_{2} & =z_{3}+w_{2}^{\rho_{2}} \\
\dot{z}_{3} & =z_{4} \\
\dot{z}_{4} & =z_{3} z_{4}
\end{aligned}
$$

with $h_{i}=w_{i}^{1}, 1 \leq i \leq 3$, and $n-k=2(m-1)=4$. It is immediate that for the above system we can always construct vector fields $g_{1}, g_{2}$ and $g_{3}$ such that $\left(\Sigma_{c}, h\right)$ is flat of 
differential weight $n+m+p^{*}=n+m+2$, which is the minimal possible, and given by:

$$
\begin{aligned}
& \dot{w}_{1}^{j}=w_{1}^{j+1} \\
& \dot{w}_{1}^{\rho_{1}}=a_{1}(w)+u_{1} \\
& \begin{aligned}
\dot{w}_{2}^{j} & =w_{2}^{j+1} \\
\dot{w}_{2}^{\rho_{2}} & =a_{2}(w)+z_{2}^{1} u_{1}
\end{aligned} \\
& \dot{w}_{3}^{j}=w_{3}^{j+1} \\
& \dot{w}_{3}^{\rho_{3}}=a_{3}(w)+z_{3}^{1} u_{1} \\
& \begin{array}{l}
\dot{z}_{2}^{1}=z_{2}^{2} \\
\dot{z}_{2}^{2}=z_{3}^{1}+w_{2}^{\rho_{2}}+u_{2}
\end{array} \\
& \begin{array}{l}
\dot{z}_{3}^{1}=z_{3}^{2} \\
\dot{z}_{3}^{2}=z_{3}^{1} z_{3}^{2}+u_{3} .
\end{array}
\end{aligned}
$$

This is, in fact, the normal form NF1, for which we have chosen $\psi_{2}=z_{2}^{1}=z_{1}, \psi_{3}=z_{3}^{1}=$ $z_{3}$, with $\ell=1$ (i.e., there is only one $z$-block, so the upper index $s$ has been omitted) and all $z$-chains of length equal to $p=2$ (yielding the minimal possible differential weight). Notice that $2 m-1=5$ equations have been modified, so the above system can be seen as an optimal normal form NF1. Namely, its differential weight $n+m+2$ is minimal and the number of controls $u_{i}$ affecting the originally unobserved $z$-subsystem is two (maximal). So it is, actually, the consistent normal form $\mathrm{NF}_{\text {min }}^{*}$ of Theorem 3.3 given in Section 3.3 below.

On the other hand, it is easy to see that the function $\psi(w, z)=z_{1}$ verifies Theorem 3.2 (M1) around any $\left(w_{0}, z_{0}\right)$, and thus we can construct another flat control system (of differential weight greater than $n+m+2$ ) for which the vector fields $g_{1}, g_{2}$ and $g_{3}$ have together $m+1=4$ nonzero components only. First, introduce new coordinates $z^{q}=L_{f}^{q-1} \psi, 1 \leq q \leq 4$, that transform the unobserved subsystem into $\dot{z}^{q}=z^{q+1}$, $1 \leq q \leq 3, \dot{z}^{4}=b^{4}(w, z)$, where $b^{4}(w, z)=\left(z^{3}-w_{2}^{\rho_{2}}\right)\left(z^{4}-a_{2}(w)\right)+L_{f} a_{2}(w)$. For that form, we construct

$$
\begin{array}{rlrl}
\dot{w}_{i}^{j}=w_{i}^{j+1}, 1 \leq j \leq \rho_{i}-1, & & \dot{w}_{3}^{j}=w_{3}^{j+1}, 1 \leq j \leq \rho_{3}-1, \\
\dot{w}_{i}^{\rho_{i}}=a_{i}(w)+u_{i}, & & \dot{w}_{3}^{\rho_{3}}=a_{3}(w)+z^{1} u_{1}, \\
& 1 \leq i \leq 2, & \dot{z}^{q}=z^{q+1}, 1 \leq q \leq 3, \\
& \dot{z}^{4}=b^{4}(w, z)+u_{3},
\end{array}
$$

which is obviously flat with $h=\left(w_{1}^{1}, w_{2}^{1}, w_{3}^{1}\right)$ being a flat output of differential weight at least $n+m+4$ since we necessarily have to differentiate $u_{1}$ four times, and depending on the function $a_{3}(w)$, the control $u_{2}$ may also need to be differentiated (at most three times, so the differential weight of $h$ cannot exceed $n+m+7)$.

\subsubsection{When is $\left(\mathrm{NF}_{\min }, h\right)$ of minimal differential weight?}

First, consider the case $m=2$ (i.e., we have only two outputs $h_{1}$ and $h_{2}$ and the constructed flat control system will be a two-input control-affine system). The $z$-dimension $n-k$ can be any. Since $m-1=1$, we have $n-k=p \geq 1, r=0, p=p^{*}$, and the minimal differential weight of $h$ as a flat output of $\Sigma_{c}$ is $n+m+p^{*}=n+2+(n-k)$. Assume that a function $\psi$ satisfying Theorem 3.2 (M1) exits around $x_{0}$ (if $x_{0}$ is not an equilibrium, by item (M2), $\psi$ always exists). Then $\mathrm{NF}_{\text {min }}$ can be constructed and is given by

$$
\begin{cases}\dot{w}_{1}^{j}=w_{1}^{j+1}, & \dot{w}_{2}^{j}=w_{2}^{j+1}, \\ \dot{w}_{1}^{\rho_{1}}=a_{1}(w)+u_{1}, & \dot{w}_{2}^{\rho_{2}}=a_{2}(w)+z^{1} u_{1}, \\ & \dot{z}^{q}=z^{q+1} \\ & \dot{z}^{n-k}=b^{n-k}(w, z)+u_{2},\end{cases}
$$

where $1 \leq j \leq \rho_{i}-1,1 \leq i \leq 2,1 \leq q \leq n-k-1$, and $h=\left(w_{1}^{1}, w_{2}^{1}\right)$ is of differential weight $n+m+p^{*}=n+2+(n-k)$, which is the minimal possible.

Observe that in the case $m=2$, no matter whether we construct $\left(\Sigma_{c}, h\right)$ as $(\mathrm{NF} 1, h)$, $(\mathrm{NF} 2, h)$ or $\left(\mathrm{NF}_{\min }, h\right)$, the differential weight of $h$ is always minimal, equal to $n+$ 
$2+(n-k)$, although the three forms, in general, do not coincide. If, however, in the construction of NF1, we choose the first $\mathbb{R}$-valued (there is only one since $m-1=1$ ) dummy output as $\psi_{2}^{1}=\psi$, with $\psi$ satisfying Theorem $3.2(\mathrm{M} 1)$, then $\left(\Sigma, h^{1}\right)$ is locally observable and the resulting control system is in the form $(22)$, that is, NF1 and $\mathrm{NF}_{\text {min }}$ coincide and the minimal differential weight of $h$ as a flat output of both of them is $n+m+p^{*}=n+m+(n-k)$.

Let us now consider the case when $m$ is any but $m \geq 3$, and $\operatorname{dim} z=n-k=1$ (i.e., $z=z_{1}$ and is the only variable that cannot be observed with the original output $h$ ) implying that $p=0$ and $r=1$, thus $p^{*}=1$ and the minimal differential weight of $h$ as a flat output of $\Sigma_{c}$ is $n+m+p^{*}=n+m+1$. If $\operatorname{dim} z=1$, then a function $\psi$ satisfying Theorem 3.2 (M1) always exists, independently whether $x_{0}$ is an equilibrium or not (simply take any arbitrary function $\psi(w, z)$ such that $\mathrm{d} \psi\left(w_{0}, z_{0}\right) \notin \mathcal{H}\left(w_{0}, z_{0}\right)$, i.e., $\frac{\partial \psi}{\partial z}\left(w_{0}, z_{0}\right) \neq 0$, and put $\left.z^{1}=\psi(x)\right)$. Hence $\mathrm{NF}_{\min }$ can always be constructed and is given by

$$
\left\{\begin{array}{rlrl}
\dot{w}_{i}^{j}= & w_{i}^{j+1}, 0 \leq j \leq \rho_{i}-1, & \dot{w}_{m}^{j} & =w_{m}^{j+1}, 0 \leq j \leq \rho_{m}-1, \\
\dot{w}_{i}^{\rho_{i}}= & a_{i}(w)+u_{i}, & \dot{w}_{m}^{\rho_{m}} & =a_{m}(w)+z^{1} u_{1}, \\
1 \leq i \leq m-1, & \dot{z}^{1} & =b^{1}\left(w, z^{1}\right)+u_{m} .
\end{array}\right.
$$

The differential weight of $h$ as a flat output of (23) is $n+m+p^{*}=n+m+1$ which is the minimal possible. Observe that system (23) is also in the form NF3. In fact, in the case $n-k=1$, the forms NF3 and $\mathrm{NF}_{\min }$ actually coincide and the minimal differential weight of $h$ as a flat output of both of them is $n+m+p^{*}=n+m+1$.

To sum up, if $n-k=1$ (independently of the value of $m$ ) or $m=2$ (independently of the value of $n-k)$, the output $h$ as a flat output of $\Sigma_{c}$ is always of minimal differential weight $n+m+p^{*}$ no matter whether $\left(\Sigma_{c}, h\right)$ is constructed as (NF1, $\left.h\right),(\mathrm{NF} 2, h),(\mathrm{NF} 3, h)$ or $\left(\mathrm{NF}_{\min }, h\right)$. In all other cases, if there exists a function $\psi$ verifying Theorem 3.2 (it always exists if $x_{0}$ is not an equilibrium), flat inputs that minimally modify the original dynamical system can always be constructed and the corresponding flat control system is given by $\left(\mathrm{NF}_{\min }, h\right)$, but the differential weight of $h$ as a flat output of $\mathrm{NF}_{\text {min }}$ is always greater than that of the (in general, nonequivalent) flat control systems obtained either with $(\mathrm{NF} 1, h)$ or $(\mathrm{NF} 2, h)$, if $n-k \geq m-1$, or with $(\mathrm{NF} 3, h)$, if $n-k<m-1$.

\subsubsection{The role of the $z$-subsystem and of the flat inputs affecting it for the application to private communication}

Observe again that, contrary to the forms of [20] whose $z$-subsystems are affected by $m-1$ (respectively, $r$ if $r=n-k<m-1$ ) inputs, one control only appears in the $z$-part of $\mathrm{NF}_{\min }$. Furthermore, when expressing all inputs in terms the flat output $h$ and its successive derivatives, the $z$-subsystem of $\mathrm{NF}_{\min }$ is used for calculating $u_{m}$ only $\left(u_{1}, \ldots, u_{m-1}\right.$ being expressed with the help of the $w$-subsystem only). This remark is extremely important for applications of flat inputs to private communication (see [21, 2, 30]).

For private communication, the flat inputs $u_{1}, \ldots, u_{m}$ correspond to confidential messages to be sent to the receiver, $(\Sigma, h)$ is the transmitter to which $\sum_{i=1}^{m} u_{i} g_{i}$ is added in such a way that the obtained $\left(\Sigma_{c}, h\right)$ is flat. Both the sender and receiver know completely the equations of the flat control system $\Sigma_{c}$ and in order to transmit a message $u(t)=\left(u_{1}(t), \ldots, u_{m}(t)\right)$, the sender chooses an initial condition $x_{0}$ and calculates the corresponding output signal $y(t)=h(x(t, u(t)))$, where $x(t, u(t))$ is the solution corresponding to $u(t)$, and transmits it via the communication multiplexed channel. The 
receiver receives the signal $y(t)$ and knowing the flat system $\Sigma_{c}$ calculates $u(t)$ as a function of $y(t)$ and its successive time-derivatives.

In the context of private communication, the unobserved $z$-subsystem plays an important role for increasing the safety. Indeed, if we consider the system $\left(\Sigma_{c}, h\right)$ in $(w, z)$ coordinates, the messages that affect the $w$-part can be expressed with the help of the $w$-variables only (we never use the linking terms) and thus the degree of security is significantly lower than for those affecting the $z$-part. Therefore the $z$-subsystem can be seen as a second level of security. Moreover, in order to decode the messages associated to the $z$-part, we always have to decode beforehand those affecting the $w$-subsystem. So for private communication, we would like to have a minimal number of different inputs involved in the $w$-equations (they correspond to the confidential messages with a lower security degree) and thus, a maximal number of different inputs affecting the $z$-subsystem (they correspond to the confidential messages with a higher security degree). The minimal number of controls appearing in the $w$-part is either one, if $n-k \geq m-1$ (and this is the case when the flat control system is constructed as $(\mathrm{NF} 1, h)$ or as $(\mathrm{NF} 2, h))$, or $m-r$, if $n-k=r<m-1$ (corresponding to the case when a flat control system is constructed as $(\mathrm{NF} 3, h)$ ).

So, for the solution proposed in [20], the number of different flat inputs involved in the $w$-subsystem is minimal and moreover, the $w$-part is minimally modified by adding flat inputs (only $m$ equations involve the control and we have seen that we cannot achieve flatness by modifying less than $m$ w-equations). The $w$-subsystem of $\mathrm{NF}_{\min }$ also possesses $m$ modified equations, however the number of inputs affecting it is maximal, equal to $m-1$. On the other hand, as discussed in Section 3.2.1. the differential weight of $\left(\mathrm{NF}_{\min }, h\right)$ is always (except the two cases, $m=2$ or $n-k=1$, of Section 3.2.2) greater than that of the flat control systems obtained either with $(\mathrm{NF} 1, h)$ or $(\mathrm{NF} 2, h)$ or $(\mathrm{NF} 3, h)$. Given this remark, two natural questions arise:

Question 3.3. What is the minimally modified construction consistent with the minimal differential weight $n+m+p^{*}$ and the minimal number of different controls affecting the $w$-equations (one control, if $n-k \geq m-1$, or $r$ controls, if $n-k=r<m-1$ )?

Question 3.4. How to choose the corresponding linking terms leading to such a modification?

\subsection{Flat inputs construction for a consistent minimal mod- ification}

Remark 3.9 (*-Minimal modification). From now on, when we say that a system is *-minimally modified (notice the use of the asterisk symbol), we refer to a minimal modification consistent with the minimal differential weight $n+m+p^{*}$ and the minimal number of different controls affecting the $w$-part (one control, if $n-k \geq m-1$, or $r$ controls, if $n-k=r<m-1$ ).

When constructing a $*$-minimally modified flat control system in the case $n-k \geq$ $m-1$, the best we can hope for is that only $2 m-1$ equations involve the input. Indeed, we necessarily have one for each $w_{i}$-chain involving one distinguished control, say $u_{1}$, and $m-1$ equations for the $z$-subsystem that has to be affected by the remaining inputs $u_{i}$, for $2 \leq i \leq m$. Similarly, it is easy to see that if $n-k=r<m-1$, the best we can hope for is $m+r *$-minimally modified equations.

Remark 3.10 (When are the constructions of [20 *-minimally modified?). Notice that if $n-k=m-1$ (i.e., $\operatorname{dim} z=m-1$ ), when constructing the flat control system either 
with $(\mathrm{NF} 1, h)$ or with $(\mathrm{NF} 2, h)$, all $z$-chains are of length one and we always obtain a *-minimally modified system containing only $2 m-1$ equations involving the input. In this case, the only difference between both forms is that for NF2 the drift of the $z$-part is rectified (i.e., $\dot{z}_{2}^{1}=1+u_{2}$ and $\dot{z}_{i}^{1}=u_{i}$, for $3 \leq i \leq m$ ), while for NF1, it can be any (i.e., $\dot{z}_{i}^{1}=b_{i}^{1}(w, z)+u_{i}$, for $2 \leq i \leq m$, where $b_{i}^{1}$ are arbitrary functions). Finally, observe that the flat control system (NF3, $h$ ), corresponding to the case $n-k=r<m-1$, is always *-minimally modified.

Therefore, from now on, we suppose that $n-k>m-1$ (so, in particular, we have $p \geq 1$ and following [20], $\left(\Sigma_{c}, h\right)$ may be constructed either as $(\mathrm{NF} 1, h)$ or as $\left.(\mathrm{NF} 2, h)\right)$. For both forms NF1 and NF2, the $w$-equations are minimally modified (and moreover, involve $u_{1}$ only, the modification being consistent with the minimal number of different controls affecting the observed $w$-part) but the $z$-subsystem of NF2 is maximally modified since the control appears in each $z$-equation. Thus, if we want to obtain a $*$-minimally modified flat control system $\Sigma_{c}$, then we necessarily have to construct $\Sigma_{c}$ using the procedure of the normal form NF1. Recall that NF1 is constructed by completing the original output $h$ with dummy outputs (linking terms) in order to achieve observability with respect to the new output. The question is actually how to chose the linking terms for which the $z$-part is also minimally modified. The following theorem answers it as well as the related Questions 3.3 and 3.4 above.

Theorem 3.3 states that the existence and construction of a $*$-minimally modified system is related to the existence of an $(m-1)$-tuple of dummy outputs $\psi=\left(\psi_{2}, \ldots, \psi_{m}\right)$ completing $h$ to $h^{1}=(h, \psi)$ that renders the system $\Sigma$ observable at $x_{0}$ with well chosen OQI's. Around an equilibrium $x_{0}$ of $f$, this problem reduces, actually, to a linear one, as asserted by item (M3)* of Theorem 3.3 below. The same notations as those of Theorem 3.2 (M3), treating the case of an equilibrium point $x_{0}$ (in particular, the matrix $A$ defined by (8) just before the statement of Theorem 3.2), will be used in conditions $(\mathrm{M} 3)^{*}$ and $(\mathrm{M} 4)^{*}$ below.

Theorem 3.3. Consider the observed system $(\Sigma, h)$ around any $x_{0} \in \mathcal{X}$ and let $\left(\rho_{1}, \ldots, \rho_{m}\right)$ be POQI's at $x_{0}$.

$(M 1)^{*}$ Suppose that there exists an $(m-1)$-tuple of functions $\left(\psi_{2}, \ldots, \psi_{m}\right)$ completing $h$ to $h^{1}=\left(h_{1}, \ldots, h_{m}, \psi_{2}, \ldots, \psi_{m}\right)$ such that $\left(f, h^{1}\right)$ is observable at $x_{0}$ and admits OQI's $\left(\rho_{1}, \ldots, \rho_{m}, \nu_{2}, \ldots, \nu_{m}\right)$ at $x_{0}$. Then there exist local coordinates $(w, z)$, with $w$ given by (4) and $z_{i}^{q}=L_{f}^{q-1} \psi_{i}(x)$, for $1 \leq q \leq \nu_{i}$, bringing $(\Sigma, h)$ into (5)-(6) with the unobserved subsystem (6) given by:

$$
\left\{\begin{array}{l}
\dot{z}_{i}^{q}=z_{i}^{q+1}, \quad 1 \leq q \leq \nu_{i}-1 \\
\dot{z}_{i}^{\nu_{i}}=b_{i}(w, z), \quad 2 \leq i \leq m
\end{array}\right.
$$

For this form, we can always locally construct $g_{1}, \ldots, g_{m}$ that have together $2 m-1$ nonzero components, and such that $\left(\Sigma_{c}, h\right)$ is $x$-flat at $x_{0}$ and given by the following form:

$$
N F_{\text {min }}^{*}\left\{\begin{array}{rlrl}
\dot{w}_{1}^{j}=w_{1}^{j+1}, & \dot{w}_{i}^{j}=w_{i}^{j+1}, \\
\dot{w}_{1}^{\rho_{1}}=a_{1}(w)+u_{1}, & \dot{w}_{i}^{\rho_{i}}=a_{i}(w)+z_{i}^{1} u_{1}, \\
& \dot{z}_{i}^{q}=z_{i}^{q+1}, \\
& \dot{z}_{i}^{\nu_{i}}=b_{i}(w, z)+u_{i}, \\
& & 2 \leq i \leq m,
\end{array}\right.
$$

where $1 \leq j \leq \rho_{i}-1,1 \leq q \leq \nu_{i}-1$, and $h=\left(w_{1}^{1}, \ldots, w_{m}^{1}\right)$ is a flat output of differential weight at least $n+m+\max _{2 \leq i \leq m} \nu_{i}$. 
(M2)* If $f\left(x_{0}\right) \neq 0$, then an $(m-1)$-tuple of functions $\left(\psi_{2}, \ldots, \psi_{m}\right)$ satisfying the conditions of $(M 1)^{*}$ always exists and therefore we can always construct the modified flat control system $N F_{\text {min }}^{*}$.

$(M 3)^{*}$ If $f\left(x_{0}\right)=0$, then the existence of the $(m-1)$-tuple of functions $\left(\psi_{2}, \ldots, \psi_{m}\right)$ satisfying $(M 1)^{*}$ is equivalent to the existence of a matrix $C \in \mathbb{R}^{(m-1) \times(n-k)}$ such that

$$
\operatorname{rk}\left(\begin{array}{c}
C \\
C A \\
\vdots \\
C A^{n-k-1}
\end{array}\right)=n-k,
$$

in which case a solution of $(M 1)^{*}$ is $\left(\psi_{2}(w, z), \ldots, \psi_{m}(w, z)\right)=C z$.

$\left(M_{4}\right)^{*}$ The differential weight of $h$ as a flat output of $N F_{\text {min }}^{*}$ is the minimal possible $n+$ $m+p^{*}$ if and only if the integers $\nu_{i}$ satisfy (after permuting $\psi_{i}$ 's, if necessary) $\nu_{i}=p+1$, for $2 \leq i \leq r+1$, and $\nu_{i}=p$, for $r+2 \leq i \leq m$. Moreover an $(m-1)$-tuple of functions $\left(\psi_{2}, \ldots, \psi_{m}\right)$, satisfying both $(M 1)^{*}$ and the latter condition, always exists if $f\left(x_{0}\right) \neq 0$, while for $f\left(x_{0}\right)=0$, it exists if and only if there exists a matrix $C \in \mathbb{R}^{(m-1) \times(n-k)}$ such that

$$
\operatorname{rk}\left(\begin{array}{c}
C \\
C A \\
\vdots \\
C A^{p^{*}-1}
\end{array}\right)=n-k .
$$

Proof. The proof, based on the following algorithm, is given in Section 5.3 .

\subsubsection{Algorithm for constructing $g_{1}, \ldots, g_{m}$ of the normal form $\mathbf{N F}_{\min }^{*}$}

Consider the system $(\Sigma, h)$ around any $x_{0} \in \mathcal{X}$, so $(\Sigma, h)$ admits POQI's $\left(\rho_{1}, \ldots, \rho_{m}\right)$ at $x_{0}$. Suppose that there exists an $(m-1)$-tuple of smooth functions $\left(\psi_{2}, \ldots, \psi_{m}\right)$ satisfying condition (M1)*. By introducing, around $x_{0}$, the $w$-coordinates given by (4) and the $z$-coordinates $z_{i}^{q}=L_{f}^{q-1} \psi_{i}$, for $1 \leq q \leq \nu_{i}, 2 \leq i \leq m$, the system $(\Sigma, h)$ is brought into the observed-unobserved form (5)-(6) with the unobserved subsystem being of the form (24). For that form define the control vector fields:

$$
g_{1}=\frac{\partial}{\partial w_{1}^{\rho_{1}}}+\sum_{i=2}^{m} z_{i}^{1} \frac{\partial}{\partial w_{i}^{\rho_{i}}} \quad \text { and } \quad g_{i}=\frac{\partial}{\partial z_{i}^{\nu_{i}}}, 2 \leq i \leq m .
$$

that have together $2 m-1$ nonzero components. The associated control system is in the form $\mathrm{NF}_{\text {min }}^{*}$ which is $x$-flat at $x_{0}$ with $h=\left(w_{1}^{1}, w_{2}^{1}, \ldots, w_{m}^{1}\right)$ being a flat output of differential weight at least $n+m+\max _{2 \leq i \leq m} \nu_{i} \cdot \triangleleft$

The above definition provides an explicit construction of the control vector fields $g_{i}$.

Proposition 3.2. Consider the observed dynamical system $(\Sigma, h)$ around $x_{0}$ and suppose that a $(m-1)$-tuple of functions $\left(\psi_{2}, \ldots, \psi_{m}\right)$ satisfies the assumption of Theorem 3.3 $(M 1)^{*}$. Denote $\psi_{i}^{q}=L_{f}^{q-1} \psi_{i}$, for $1 \leq q \leq \nu_{i}, 2 \leq i \leq m$, and $\varphi_{i}^{j}=L_{f}^{j-1} h_{i}$, for $1 \leq j \leq \rho_{i}, 1 \leq i \leq m$. Vector fields $g_{1}, \ldots, g_{m}$ such that the $x$-flat control system $\left(\Sigma_{c}, h\right)$ is $*$-minimally modified can be constructed as follows: 
- for $g_{1}$ by

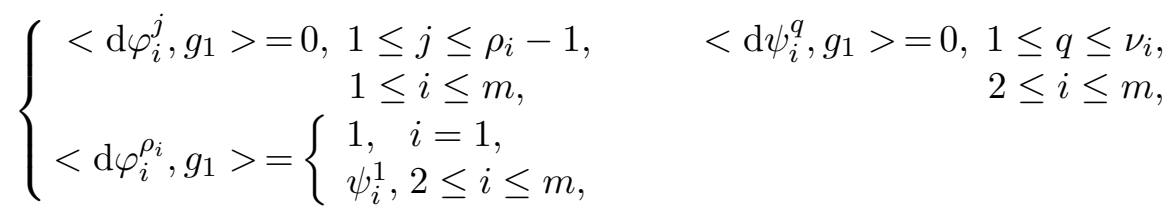

- for $g_{2}, \ldots, g_{m}$ by

$$
\left\{\begin{array}{c}
<\mathrm{d} \varphi_{i}^{j}, g_{l}>=0,1 \leq j \leq \rho_{i}, \quad<\mathrm{d} \psi_{i}^{q}, g_{l}>=\left\{\begin{array}{l}
0,1 \leq q \leq \nu_{i}-1, \\
\delta_{i l}, q=\nu_{i},
\end{array}\right. \\
1 \leq i \leq m,
\end{array}\right.
$$

where $2 \leq l \leq m$ and $\delta_{i l}=1$, if $i=l$, and 0 , otherwise.

Corollary 3.4. In a local coordinate system $x=\left(x_{1}, \ldots, x_{n}\right)$, form the $n \times n$-matrix $H^{1}(x)=\left(\begin{array}{c}H(x) \\ \Psi(x)\end{array}\right)$ whose first $k$ rows $H(x)$ are the differentials $\mathrm{d} \varphi_{i}^{j}(x)=\mathrm{d} L_{f}^{j-1} h_{i}(x)$ and the last $n-k$ rows $\Psi(x)$ are the differentials $\mathrm{d} \psi_{i}^{q}=\mathrm{d} L_{f}^{q-1} \psi_{i}$, where we use the notations of Proposition 3.2. Then equations 28)-29) are equivalent to

$$
H^{1}(x) \cdot g(x)=D(x)
$$

where $g(x)=\left(g_{1}(x) \ldots g_{m}(x)\right)$, and $D(x)=\left(d_{i}^{s}(x)\right)$, with $d_{i}^{s}$, for $1 \leq s \leq n, 1 \leq$ $i \leq m$, denoting suitable terms of the right hand-side of (28)-(29). If the $(m-1)$ tuple of functions $\left(\psi_{2}, \ldots, \psi_{m}\right)$ satisfies the assumption of Theorem $3.3(M 1)^{*}$, then the matrix $H^{1}(x)$ is invertible around $x_{0}$ and

$$
g(x)=\left(H^{1}(x)\right)^{-1} \cdot D(x) .
$$

Proof. The proofs of Proposition 3.2 and Corollary 3.4 follow directly from the algorithm in Section 3.3.1 for constructing $g_{1}, \ldots, g_{m}$ of $\mathrm{NF}_{\min }^{*}$.

\subsubsection{Discussion of $\mathrm{NF}_{\text {min }}^{*}$}

Condition (M1)* actually says that if we want to construct a flat control system with a minimal modification of the $z$-equations (that is only $m-1$ modified equations), then the modified equations are necessarily those at the bottom of each $z_{i}$-chain (that have to involve the remaining inputs $u_{2}, \ldots, u_{m}$ ). Therefore, we necessarily have to use one (and only one!) $(m-1)$-tuple of linking terms $\left(z_{2}^{1}, \ldots, z_{m}^{1}\right)=\left(\psi_{2}, \ldots, \psi_{m}\right)$ such that the new output $h^{1}=\left(h_{1}, \ldots, h_{m}, \psi_{2}, \ldots, \psi_{m}\right)$ renders the original system locally observable. In that case, we obtain the normal form $\mathrm{NF}_{\text {min }}^{*}$ which can be seen as a particular case of NF1 for which $\ell=1$.

If $x_{0}$ is not an equilibrium (i.e., $f\left(x_{0}\right) \neq 0$ ), then, according to $(\mathrm{M} 2)^{*}$, such linking terms always exist and lead to a control system for which the vector fields $g_{1}, \ldots, g_{m}$ distort $2 m-1$ equations only (the modification being consistent with the minimal number of different controls affecting the $w$-part). If $f\left(x_{0}\right)=0$, the problem of finding $\left(\psi_{2}, \ldots, \psi_{m}\right)$ reduces to a linear problem, see (M3)*

On the other hand, the differential weight of $h$ as a flat output of $N F_{\min }^{*}$ is at least $n+m+\max _{2 \leq i \leq m} \nu_{i}$. Indeed, the inputs $u_{2}, \ldots, u_{m}$ appear at the bottom level of each $z_{i}$-chain, and it follows that, in the best case, $u_{1}$ is the only input that needs to be differentiated in order to express all states and controls with the help the $h_{i}$ 's and their time-derivatives. For each $z_{i}$-chain, the input $u_{1}$ needs to be differentiated $\nu_{i}$ times. 
Hence, in the best case, the differential weight of $h$ is $n+m+\max _{2 \leq i \leq m} \nu_{i}$. In some cases, we may need additionally to differentiate other inputs among $u_{2}, \ldots, u_{m}$. This is similar to the differential weight of $\left(\mathrm{NF}_{\min }, h\right)$, see Section 3.1.2. The condition for $\left(\mathrm{NF}_{\min }^{*}, h\right)$ to be of minimal possible differential weight $n+m+p^{*}$ is actually that all $z_{i^{-}}$ chains are of length either $p$ or $p+1$, see $(\mathrm{M} 4)^{*}$, (this reminds very much the form NF2) and again, according to $(\mathrm{M} 4)^{*}$, linking terms assuring the minimal differential weight always exit if the nominal point around which we work is not an equilibrium. Around an equilibrium $x_{0}$, they exists if we can find a dummy output $C z$ such that all observability indices of $(A, C)$, where $A=\frac{\partial b}{\partial z}\left(x_{0}\right)$, are equal either to $p$ or $p+1$. Notice that around an equilibrium, there are systems (e.g., systems such that $A=\frac{\partial b}{\partial z}\left(x_{0}\right)=0$ ) for which we cannot construct flat inputs in an optimal way (leading to a flat control system of the form either $\left(\mathrm{NF}_{\min }, h\right)$ or $\left.\left(\mathrm{NF}_{\min }^{*}, h\right)\right)$, but we can always compute them using the procedure of 20. Finally, observe that similarly to $\left(\mathrm{NF}_{\text {min }}, h\right)$, see Remark 3.4, any $u=\left(u_{1}, u_{2}, \ldots, u_{m}\right)$ for which $u_{1}=0$ creates flatness singularities in the control space for $\left(\mathrm{NF}_{\min }^{*}, h\right)$.

An interesting and important observation is that in many cases, we can obtain from the optimal forms $\left(\mathrm{NF}_{\min }, h\right)$ or $\left(\mathrm{NF}_{\min }^{*}, h\right)$ a feedback equivalent flat control system with $m$ modified equations only, as stated by the following corollary.

Corollary 3.5. Suppose $n-k \geq 2$. If there exists either a function $\psi$ satisfying Theorem 3.2 (M1) or a $(m-1)$-tuple $\left(\psi_{2}, \ldots, \psi_{m}\right)$ satisfying Theorem 3.3 (M1)* with $\nu_{i} \geq 2$, for $2 \leq i \leq m$, then there always exists a construction that renders the system flat and modifies $m$ equations only.

\section{Proof. See Section 5.4 .}

The main idea leading to Corollary 3.5 is to rectify, if possible, the control vector field $g_{1}$ of $\left(\mathrm{NF}_{\text {min }}, h\right)$ or $\left(\mathrm{NF}_{\min }^{*}, h\right)$ respectively (the control vector field $g_{2}, \ldots, g_{m}$ of those forms are already rectified and each of them has only one nonzero component) or, equivalently, to apply a suitable change of coordinates mixing the observed $w$-variables and the unobserved $z$-variables. As explained in Remark 3.3 , the distribution $\mathcal{G}=\operatorname{span}\left\{g_{1}, \ldots, g_{m}\right\}$ is not known a priori, and first, it has to be constructed in an optimal way with the help of $\left(\mathrm{NF}_{\min }, h\right)$ or $\left(\mathrm{NF}_{\min }^{*}, h\right)$, and only then, if $\mathcal{G}$ is involutive (which is the case if all $z_{i}$-chains are of length at least 2 ), it can be rectified leading to a flat control system with $m$ equations involving the flat inputs (but for which there is no longer a splitting between the observable $w$-subsystem and the unobserved $z$-subsystem).

To summarize, if, the goal is to modify the original dynamics (5)-(6) in the simplest possible way (without asking for $h$ to be of minimal differential weight, or imposing any constraints on the number of flat inputs that are added to the observed $w$-part), then $\mathrm{NF}_{\text {min }}$ is such a construction. If, on the other hand, it is important to obtain a flat control system with the differential weight of $h$ being the minimal possible, or with a minimal number of inputs affecting the $w$-subsystem, then $\mathrm{NF}_{\text {min }}^{*}$ for the case $n-k \geq m-1$, respectively, NF3 for $n-k<m-1$, provide such a construction.

\subsection{Global results and *-minimal modification of $\Sigma$}

All normal forms presented in this paper are local and valid around any point of an open and dense subset $\mathcal{X}$ of $\mathbb{X}$ guaranteeing a generic construction of the vector fields $g_{1}, \ldots, g_{m}$. Under some hypothesis, $g_{1}, \ldots, g_{m}$ may however exist globally on $\mathbb{X}$ and the control system may be $x$-flat on $\mathbb{X}$ with the flat output being globally defined as $\varphi_{i}=h_{i}$, 
$1 \leq i \leq m$, (but, with representation (2) which is, in general, local, see [20] where this is illustrated by an example). The following proposition gives conditions allowing a global construction of $g_{1}, \ldots, g_{m}$ leading to a $*$-minimally modified control system. Moreover, it asserts that under those conditions, the manifold $\mathbb{X}$ has to be of a special form (see [12] for the notion of parallelizable manifold used in item (ii) below).

Proposition 3.3. Consider the observed system $(\Sigma, h)$ on $\mathbb{X}$. The following hold:

(i) Suppose that $(\Sigma, h)$ admits uniform POQI's $\left(\rho_{1}, \ldots, \rho_{m}\right)$ on $\mathbb{X}$. If there exist functions $\left(\psi_{2}, \ldots, \psi_{m}\right)$ globally defined on $\mathbb{X}$ satisfying condition $(M 1)^{*}$ of Theorem 3.3 around any $x_{0} \in \mathbb{X}$ with uniform $O Q I$ 's $\left(\rho_{1}, \ldots, \rho_{m}, \nu_{2}, \ldots, \nu_{m}\right)$ on $\mathbb{X}$, then there exist $g_{1}, \ldots, g_{m}$ globally defined on $\mathbb{X}$ that lead to $\left(N F_{\min }^{*}, h\right)$ and have together $2 m-1$ number of nonzero components. The obtained control system $\left(N F_{\min }^{*}, h\right)$ is locally $x$-flat around any $x_{0} \in \mathbb{X}$ and moreover, if each $\nu_{i}$ is either $p$ or $p+1$, then the differential weight of $h$ as a flat output of $N F_{\min }^{*}$ is $n+m+p^{*}$ and the system is *-minimally modified.

(ii) If $(\Sigma, h)$ on $\mathbb{X}$ satisfies the condition of item (i) above, then $\mathbb{X}$ is parallelizable and $\mathbb{X}$ is either open (i.e., non compact) or compact and in that case, $\mathbb{X}$ is diffeomorphic to the $n$-dimensional torus $\mathbb{T}^{n}=\mathbb{S}^{1} \times \cdots \times \mathbb{S}^{1}$.

Proof. The proof is given is Section 5.5 .

Observe that if POQI's are uniform on $\mathbb{X}$, then the rank of the codistribution $\mathcal{H}$ is constant everywhere. It is clear that under Proposition 3.3. the functions $w_{i}^{j}=L_{f}^{j-1} h_{i}$, for $1 \leq j \leq \rho_{i}, 1 \leq i \leq m$, and $z_{i}^{q}=L_{f}^{q-1} \psi_{i}(x)$, for $1 \leq q \leq \nu_{i}, 2 \leq i \leq m$, are defined globally and form around any $x_{0}$ a local system of coordinates. Therefore, the vector fields $g_{1}, \ldots, g_{m}$ are also globally constructed on $\mathbb{X}$.

The system $\left(\Sigma_{c}, h\right)$ is globally defined on $\mathbb{X}$ and is locally, around any $x_{0}$, equivalent to the normal form $\left(\mathrm{NF}_{\text {min }}^{*}, h\right)$. We do not claim however that it is globally equivalent to $\left(\mathrm{NF}_{\min }^{*}, h\right)$ unless $(w, z)=\Phi(x)$, where $w_{i}^{j}=L_{f}^{j-1} h_{i}, 1 \leq j \leq \rho_{i}$, and $z_{i}^{q}=L_{f}^{q-1} \psi_{i}$, $1 \leq q \leq \nu_{i}$, is a global diffeomorphism of $\mathbb{X}$. For the same reason, the flatness of $\left(\Sigma_{c}, h\right)$ is local around any $\left(x_{0}, u_{0}\right)$, such that $u_{10} \neq 0$, although flat output components $h_{1}, \ldots, h_{m}$ are defined globally.

\section{Example: application to private communication}

In this section we discuss from the point of view of minimal modification the solution proposed in 20] for an application to private communication. We use a transmitter, composed of two independent chaotic systems, a Lorenz circuit $\left(x_{\ell}, y_{\ell}, z_{\ell}\right)$, see, e.g., [17, and a Rössler circuit $\left(x_{r}, y_{r}, z_{r}\right)$, see, e.g., [26], of the form (the notation $(C h)$ referring to the chaotic behavior):

$$
(C h): \begin{cases}\dot{x}_{\ell}=\sigma\left(y_{\ell}-x_{\ell}\right) & \dot{x}_{r}=-y_{r}-z_{r} \\ \dot{y}_{\ell}=-k_{d} x_{\ell} z_{\ell}+r x_{\ell}-y_{\ell} & \dot{y}_{r}=x_{r}+a y_{r} \\ \dot{z}_{\ell}=k_{d} x_{\ell} y_{\ell}-\beta z_{\ell} & \dot{z}_{r}=b+z_{r}\left(x_{r}-c\right),\end{cases}
$$

where the parameters $a, b, c, \beta, \sigma, r$ and $k_{d}$ are constant. Two messages $u_{1}(t)$ and $u_{2}(t)$ have to be sent to the receiver and in order to transmit them, we add to $(C h)$ two control vector fields $g_{1}$ and $g_{2}$ whose controls are, resp., $u_{1}$ and $u_{2}$ :

$$
\left(C h_{c}\right): \dot{x}=f(x)+u_{1} g_{1}(x)+u_{2} g_{2}(x), y_{i}=h_{i}(x), 1 \leq i \leq 2,
$$


and the masked information transmitted via the communication multiplexed channel is $\left(y_{1}(t), y_{2}(t)\right)=\left(x_{\ell}(t), z_{\ell}(t)\right)$, where $g_{1}$ and $g_{2}$ are chosen in such a way that $\left(C h_{c}\right)$ is flat with $\varphi=\left(h_{1}, h_{2}\right)=\left(x_{\ell}, z_{\ell}\right)$ being a flat output, and $f$ is the right-hand side of $(C h)$.

It is clear that with $\left(h_{1}, h_{2}\right)=\left(x_{\ell}, z_{\ell}\right)$ only the Lorenz variables can be observed and in the observed-unobserved form (5)-(6), using the new global $w$-coordinates $w_{1}^{1}=x_{\ell}$, $w_{1}^{2}=L_{f} x_{\ell}=\sigma\left(y_{\ell}-x_{\ell}\right), w_{2}^{1}=z_{\ell}$, the Lorenz circuit is equivalently given by: $\dot{w}_{1}^{1}=w_{1}^{2}$, $\dot{w}_{1}^{2}=-\sigma k_{d} w_{1}^{1} w_{2}^{1}+\sigma(r-1) w_{1}^{1}-(\sigma+1) w_{1}^{2}=a_{1}(w)$ and $\dot{w}_{2}^{1}=k_{d} w_{1}^{1}\left(\frac{1}{\sigma} w_{1}^{2}+w_{1}^{1}\right)-\beta w_{2}^{1}=$ $a_{2}(w)$.

Notice that here, the unobserved subsystem described by the Rössler circuit is completely independent of the observed Lorenz circuit. Define the linking term $z^{1}=\psi\left(w, x_{r}, y_{r}, z_{r}\right)$ by $\psi=y_{r}$ and compute its successive time-derivatives. We get $L_{f} \psi=x_{r}+a y_{r}$ and $L_{f}^{2} \psi=-\left(y_{r}+z_{r}\right)+a\left(x_{r}+a y_{r}\right)$. It is clear that $\mathrm{d} \psi, \mathrm{d} L_{f} \psi$ and $\mathrm{d} L_{f}^{2} \psi$ are independent everywhere, and $z^{q}=L_{f}^{q-1} \psi, 1 \leq q \leq 3$, (together with the $w$-coordinates) define a global system of coordinates on $\mathbb{R}^{3} \times \mathbb{R}^{3}$. So we globally define $g_{1}=\frac{\partial}{\partial w_{1}^{2}}+z^{1} \frac{\partial}{\partial w_{2}^{1}}$ and $g_{2}=\frac{\partial}{\partial z^{3}}$ and obtain in $(w, z)$-coordinates a system in the following form:

$$
\begin{cases}\dot{w}_{1}^{1}=w_{1}^{2} & \dot{w}_{2}^{1}=a_{2}(w)+z^{1} u_{1} \\ \dot{w}_{1}^{2}=a_{1}(w)+u_{1} & \dot{z}^{1}=z^{2} \\ & \dot{z}^{2}=z^{3} \\ & \dot{z}^{3}=b_{2}(w, z)+u_{2},\end{cases}
$$

where $b_{2}(w, z)=-b-z^{2}-a z^{3}-\left(z^{3}-z^{1}+a z^{2}\right)\left(z^{2}-a z^{1}\right)$, which is flat with $\left(w_{1}^{1}, w_{2}^{1}\right)=$ $\left(x_{\ell}, z_{\ell}\right)$ being a flat output.

Notice that only one dummy output $\psi=y_{r}$ is necessary to render $(C h)$ observable, the observed $w$-subsystem is affected only by $u_{1}$, while the remaining input appears at the bottom level of the (only) $z$-chain. System $(32)$ contains $m+1=3$ modified equations (which, according to Theorem 3.2 , is the minimal possible), so it is minimally modified and it is actually in the form $\left(\mathrm{NF}_{\min }, h\right)$. Moreover, the differential weight of $h$ equals $n+m+p^{*}=n+m+3$ (for $m=2$, we simply have $p^{*}=p=n-k$ ) and, according to Theorem 3.3 , it is the minimal possible, thus system 32 is also $*$-minimally modified and represented in the form $\left(\mathrm{NF}_{\min }^{*}, h\right)$ which, as we have already discussed, can be seen as an optimal (NF1, $h$ )-form (for which only one dummy output is needed). This example is consistent with the remarks of Section 3.2.2 and perfectly illustrates the situation when all constructions $(\mathrm{NF} 1, h),\left(\mathrm{NF}_{\min }, h\right)$ and $\left(\mathrm{NF}_{\text {min }}^{*}, h\right)$ coincide. Finally, the vector fields $g_{1}$ and $g_{2}$ (and thus system (32) ) are globally defined on $\mathbb{R}^{3} \times \mathbb{R}^{3}$, thus illustrating Proposition 3.3 .

For private communication, the chaotic behavior of the transmitter $(C h)$ (depending on the values of the constant parameters) is crucial and has to be preserved by adding the modification $u_{1} g_{1}(x)+u_{2} g_{2}(x)$. In [20], we have shown, using numerical simulations, that the chaotic behavior of $(C h)$ is preserved by adding control vector fields multiplied by suitable periodic messages into the dynamics, and second, that for suitable amplitudes of the encoded messages, the messages are recovered with a good accuracy by the receiver.

\section{Proofs}

\subsection{Proof of Theorem 3.1}

Suppose that locally, around $x_{0} \in \mathcal{X}$, there exist $z$-coordinates (completing $w$-coordinates) and control vector fields $g_{1}, \ldots, g_{m}$ that have together, in the $(w, z)$-coordinates, $m$ nonzero components and render $\left(\Sigma_{c}, h\right)$ flat at $x_{0}$. It follows that in the $(w, z)$-coordinates, 
each vector field $g_{i}$ has one (and only one) nonzero component (recall that the control vector fields are supposed everywhere independent). Assume that $m^{\prime}$ equations, with $m^{\prime} \leq m$, of the $w$-subsystem are affected by the flat inputs. We rename the $w$-variables by $\left(w_{1}, \ldots w_{k}\right)$ such that among the $w$-equations only those for $\dot{w}_{i}$, for $1 \leq i \leq m^{\prime}$, involve the controls, i.e., $\left(\Sigma_{c}, h\right)$ is of the form

$$
\begin{array}{rlr}
\dot{w}_{i}=f_{i}(w)+c_{i}(w, z) u_{i}, & 1 \leq i \leq m^{\prime}, \\
\dot{w}_{i}=f_{i}(w), & m^{\prime}+1 \leq i \leq k, \\
\dot{z} & =b(w, z)+D(w, z) \bar{u}, &
\end{array}
$$

with $\left(h_{1}, \ldots, h_{m}\right)$, where $h_{l}=h_{l}(w), 1 \leq l \leq m$, a flat output, $c_{i}(w, z)$ non vanishing smooth functions, $\bar{u}=\left(u_{m^{\prime}+1}, \ldots, u_{m}\right)$, and $D(w, z)$ a full rank $(n-k) \times\left(m-m^{\prime}\right)$-matrix (containing only $m-m^{\prime}$ nonzero terms). By applying the local invertible static feedback $v_{i}=c_{i}(w, z) u_{i}, 1 \leq i \leq m^{\prime}$, and $v_{i}=u_{i}, m^{\prime}+1 \leq i \leq m$, we get

$$
\begin{array}{llrl}
\dot{w}_{i} & =f_{i}(w)+v_{i}, & 1 \leq i \leq m^{\prime}, \\
\dot{w}_{i} & =f_{i}(w), & m^{\prime}+1 \leq i \leq k, \\
\dot{z} & =b(w, z)+D(w, z) \bar{v}, &
\end{array}
$$

where $\bar{v}=\left(v_{m^{\prime}+1}, \ldots, v_{m}\right)$. From this form, it is clear that none $z$-state nor $\bar{v}$-input can be represented in terms of $h_{l}^{(j)}, j \geq 0$, contradicting the flatness assumption. It follows that the $z$-coordinates are absent implying that $(\Sigma, h)$ is locally observable at $x_{0} \in \mathcal{X}$ (and POQI's are actually OQI's).

Even if we allow $g_{i}$ 's to be dependent at some point, they are independent on an open and dense subset, on which the same proof applies, excluding flatness.

The proof of the converse implication is contained in Remark 3.2 .

\subsection{Proof of Theorem 3.2}

Proof of (M1). The proof is given by the algorithm of Section 3.1.1 following the theorem.

Proof of (M2). Consider $(\Sigma, h)$ around any $x_{0} \in \mathbb{X}$ satisfying $f\left(x_{0}\right) \neq 0$ and such that $(\Sigma, h)$ admits POQI's $\left(\rho_{1}, \ldots, \rho_{m}\right)$ at $x_{0}$. We will prove that $\Sigma$ can be locally, around $x_{0}$, transformed into (5)-(6) with the unobserved subsystem (6) given by the following chain of integrators:

$$
\begin{aligned}
\dot{z}^{q} & =z^{q+1}, \quad 1 \leq q \leq n-k-1, \\
\dot{z}^{n-k} & =1
\end{aligned}
$$

First observe that since $f\left(x_{0}\right) \neq 0$, there exist local coordinates $(w, \tilde{z})$ in a neighborhood $\mathcal{O}_{x_{0}}$ of $x_{0}$, with $w$ given by (4) and $\tilde{z}=\Psi(x)$, bringing $(\Sigma, h)$ into (5)-(6) with the unobserved subsystem (6) transformed into ([1, 22], see also [29] for an explicit construction of rectifying coordinates):

$$
\begin{aligned}
\dot{\tilde{z}}^{q} & =0, \quad 1 \leq q \leq n-k-1, \\
\dot{\tilde{z}}^{n-k} & =1 .
\end{aligned}
$$

Notice that the latter is the uncontrolled $z$-subsystem of the form NF2 of [20], with the $z$-variables labeled differently, see Section 3.2. The right hand side of (33), denoted $b(z)$, does not vanish so can be transformed into (34) (rectified) via a diffeomorphism $\tilde{z}=\phi(z)$ (which actually is a global diffeomorphism of $\mathbb{R}^{n-k}$ ). It follows that in local coordinates $(w, z)$, where $w$ is given by (4) and $\left(z^{1}, \ldots, z^{n-k}\right)=z=\phi^{-1} \circ \Psi(x)$, with $\phi^{-1} \circ \Psi$ defined on $\mathcal{O}_{x_{0}}$ since $\phi(z)$ is global on $\mathbb{R}^{n-k}$, the original system $(\Sigma, h)$ takes the form (5)-(6) with 
the unobserved subsystem (6) given by (33) (which is of the form 10 with $b^{n-k}=1$ ). In particular, the first component $z^{1}=\psi(x)$ of $z=\phi^{-1} \circ \Psi(x)$ verifies (M1). For that form we construct the vector fields $g_{1}, \ldots, g_{m}$ by $(12)$. They have together the minimal possible, that is, $m+1$, number of nonzero components. The associated control system is in the form $\mathrm{NF}_{\min }$, with $b^{n-k}=1$, which is clearly $x$-flat at $x_{0}$ with $h=\left(w_{1}^{1}, \ldots, w_{m}^{1}\right)$ a flat output of differential weight at least $n+m+(n-k)$.

Proof of (M3). Is a particular case of that of condition (M3)* of Theorem 3.3 (with the $(m-1)$-tuple of functions $\left(\psi_{2}, \ldots, \psi_{m}\right)$ being replaced by the function $\psi$, and the $(m-1) \times(n-k)$-matrix $C$ by a row vector $)$.

\subsection{Proof of Theorem 3.3}

Proof of $(M 1)^{*}$. The proof is given by the algorithm of Section 3.3.1.

Proof of (M2)*. The proof is similar to that of condition (M2) of Theorem 3.2. We first prove that $\Sigma$ can be transformed locally, around $x_{0}$, into (5)-(6) with the unobserved subsystem (6) given by the following $m-1$ chains of integrators:

$$
\begin{aligned}
& \dot{z}_{i}^{q}=z_{i}^{q+1}, \quad 1 \leq q \leq \nu_{i}, \\
& \dot{z}_{i}^{\nu_{i}}=b_{i}, \quad 2 \leq i \leq m,
\end{aligned}
$$

where

$$
\nu_{i}=\left\{\begin{array}{ll}
p, & 2 \leq i \leq m-r, \\
p+1, & m-r+1 \leq i \leq m,
\end{array} \quad \text { and } \quad b_{i}= \begin{cases}1, & i=2, \\
0, & 3 \leq i \leq m-1,\end{cases}\right.
$$

(this recalls very much the properties of the $z$-chains of the normal form NF2 of [20], see 20 in Section 3.2 .

Like in the proof of condition (M2) of Theorem 3.2 , observe that since $f\left(x_{0}\right) \neq 0$, there exist local coordinates $(w, \tilde{z})$ in a neighborhood of $x_{0}$, with $w$ given by (4) and $\tilde{z}$ of the from $\tilde{z}=\Psi(x)$, bringing $(\Sigma, h)$ into (5)-(6) with the unobserved subsystem (6) transformed into:

$$
\begin{aligned}
\dot{\tilde{z}}_{i}^{q} & =0, \quad 1 \leq q \leq \nu_{i}, \\
\dot{\tilde{z}}_{i}^{\nu_{i}} & =b_{i} \quad 2 \leq i \leq m,
\end{aligned}
$$

where the integers $\nu_{i}$ and $b_{i}$ are defined by (36). The right hand side of (35) does not vanish so can be transformed into (37) (rectified) via a diffeomorphism $\tilde{z}=\phi(z)$. It follows that in local coordinates $(w, z)$, where $w$ is given by (4) and $z=\phi^{-1} \circ \Psi(x)$, the original system $(\Sigma, h)$ takes the form (5)-(6) with the unobserved subsystem (6) given by (35) (which is of the form (24) with the integers $\nu_{i}$ and functions $b_{i}$ given by (36)). For that form we construct the vector fields $g_{1}=\frac{\partial}{\partial w_{1}^{\rho_{1}}}+\sum_{i=2}^{m} z_{i}^{1} \frac{\partial}{\partial w_{i}^{\rho_{i}}}$ and $g_{i}=\frac{\partial}{\partial z_{i}^{\nu_{i}}}$, $2 \leq i \leq m$, that have together $2 m-1$ nonzero components and lead to form $\mathrm{NF}_{\text {min }}^{*}$ which is $x$-flat at $x_{0}$ with $h=\left(w_{1}^{1}, \ldots, w_{m}^{1}\right)$ a flat output of minimal differential weight $n+m+p^{*}$. It is clear that the $(m-1)$-tuple of functions $\left(z_{2}^{1}, \ldots, z_{m}^{1}\right)=\left(\psi_{2}(x), \ldots, \psi_{m}(x)\right)$ of $z=\phi^{-1} \circ \Psi(x)$ satisfies the assumptions of (M1)* with $\nu_{i}$ being either $p$ or $p+1$.

Proof of (M3)*. Let us now suppose that $f\left(w_{0}, z_{0}\right)=0$ and bring the system into the observed-unobserved form (5)-(6) around $\left(w_{0}, z_{0}\right)$. We can always assume, without loss of generality, that $z_{0}=0$ (but not for $w_{0}$ since $h_{i}$ may not vanish at $x_{0}$ ). Take any $(m-1)$-tuple of functions $\psi=\left(\psi_{2}, \ldots, \psi_{m}\right)$ such that $\psi_{i}\left(w_{0}, 0\right)=0$, for $2 \leq i \leq m$. Around $\left(w_{0}, z_{0}\right)=\left(w_{0}, 0\right)$, via the Taylor expansion, we have:

$$
\dot{z}=A z+B\left(w-w_{0}\right)+\bar{b}(w, z),
$$


and

$$
\psi_{i}(w, z)=C_{i} z+D_{i}\left(w-w_{0}\right)+\bar{\psi}_{i}(w, z), \quad 2 \leq i \leq m,
$$

where $\bar{b}$ and $\bar{\psi}_{i}$ contain all terms of order at least two (with respect to $z$ and $w-w_{0}$ ). It is immediate to see that $L_{f}^{q} \psi_{i}=C_{i} A^{q} z+H_{q}+\bar{\Psi}_{q}$, for any $q \geq 0$, where the functions $H_{q}$ depend on $w$ only and $\Psi_{q}$ contain terms of order at least two (with respect to $z$ and $\left.w-w_{0}\right)$. Therefore $\mathrm{d} L_{f}^{q} \psi_{i}\left(w_{0}, 0\right)=\left(C_{i} A^{q}\right) \mathrm{d} z \bmod \operatorname{span}\{\mathrm{d} w\}$, for $q \geq 0$. Denote by $C$ the $(m-1) \times(n-k)$-matrix whose rows are given by the $C_{i}$ 's. From this, we deduce that the functions $\psi_{2}, \ldots, \psi_{m}$ satisfy condition (M1)* if and only if

$$
\operatorname{rk}\left(\begin{array}{c}
C \\
C A \\
\vdots \\
C A^{n-k-1}
\end{array}\right)=n-k .
$$

In particular, if there exists a matrix $C$ for which the above rank is indeed $n-k$, then the functions $\left(\psi_{2}, \ldots, \psi_{m}\right)=C z$ verify $(\mathrm{M} 1)^{*}$.

Proof of $\left(M_{4}\right)^{*}$. It is immediate that the differential weight of $h$ as a flat output of $\mathrm{NF}_{\min }^{*}$ is the minimal possible $n+m+p^{*}$ (where $p^{*}=p$, if $r=0$, and $p^{*}=p+1$, otherwise, see (19) for the definition of $p^{*}$ ) if and only if $\max _{2 \leq i \leq m} \nu_{i}=p^{*}$. From this, the definition of $p^{*}$ and also based on $\sum_{i=2}^{m} \nu_{i}=n-k$, we deduce that the integers $\nu_{i}$ of (M1)* verify (after permuting the functions $\psi_{i}$, if necessary) $\nu_{i}=p+1$, for $2 \leq i \leq r+1$, and $\nu_{i}=p$, for $r+2 \leq i \leq m$. Finally, from the equivalence stated by (M3)*, it follows that the integers $\nu_{i}$ are as above if and only if the matrix $C$ of $(\mathrm{M} 3)^{*}$ satisfies $(26)$ (recall that $n-k=p(m-1)+r)$.

\subsection{Proof of Corollary 3.5}

Suppose that there exists an $(m-1)$-tuple of smooth functions $\left(\psi_{2}, \ldots, \psi_{m}\right)$ satisfying condition (M1)* with $\nu_{i} \geq 2$, for $2 \leq i \leq m$, and construct the form $\left(\mathrm{NF}_{\min }^{*}, h\right)$. We give the proof only for this case, that corresponding to $\left(\mathrm{NF}_{\min }, h\right)$ follows exactly the same lines. By introducing the new coordinates $\xi_{i}^{\rho_{i}}=w_{i}^{\rho_{i}}-z_{i}^{1} w_{1}^{\rho_{1}}$, for $2 \leq i \leq m$, we get

$$
N F_{m} \begin{cases}\dot{w}_{1}^{j}=w_{1}^{j+1}, & \dot{w}_{i}^{j}=w_{i}^{j+1}, \\ \dot{w}_{1}^{\rho_{1}-1}=w_{1}^{\rho_{1}}, & \dot{w}_{i}^{\rho_{i}-1}=\xi_{i}^{\rho_{i}}+z_{i}^{1} w_{1}^{\rho_{1}}, \\ \dot{w}_{1}^{\rho_{1}}=\tilde{a}_{1}\left(w, \xi, z^{1}\right)+u_{1}, & \dot{\xi}_{i}^{\rho_{i}}=\tilde{a}_{i}\left(w, \xi, z^{1}\right)-z_{i}^{2} w_{1}^{\rho_{1}} \\ & \dot{z}_{i}^{q}=z_{i}^{q+1}, \\ & \dot{z}_{i}^{\nu_{i}}=b_{i}(w, z)+u_{i}, \\ & \end{cases}
$$

with $1 \leq j \leq \rho_{i}-2,1 \leq q \leq \nu_{i}-1, z^{1}=\left(z_{2}^{1}, \ldots, z_{m}^{1}\right), \xi=\left(\xi_{2}^{\rho_{2}}, \ldots, \xi_{m}^{\rho_{m}}\right)$, where $h=\left(w_{1}^{1}, \ldots, w_{m}^{1}\right)$ is a flat output of $\mathrm{NF}_{m}$ whose exactly $m$ components are modified by adding the control vector fields.

\subsection{Proof of Proposition 3.3}

Proof of (i). The proof follows exactly the same arguments as those of condition (M1)* of Theorem 3.3 (whose proof is given by the algorithm of Section 3.3.1), with the only difference that now all functions are defined globally, they form around any $x_{0}$ a local system of coordinates, and the vector fields $g_{1}, \ldots, g_{m}$ can be globally constructed by formula (31) that is global. 
Proof of (ii). The map $H^{1}: \mathbb{X} \rightarrow \mathbb{R}^{n}$ defined in Corollary 3.4 is an immersion and thus $\mathbb{X}$ is integrably parallelizable, see $[12$ for that notion. Then by the result of [12], the manifold $\mathbb{X}$ is either open or, if compact, diffeomorphic to $\mathbb{T}^{n}=\mathbb{S}^{1} \times \cdots \times \mathbb{S}^{1}$.

\section{References}

[1] V.I. Arnold. Geometrical methods in the theory of ordinary differential equations, volume 250. Springer-Verlag New York, 1988.

[2] J.-P. Barbot, M. Fliess, and T. Floquet. An algebraic framework for the design of nonlinear observers with unknown inputs. Proc. of the 46th IEEE Conference on Decision and Control (CDC), pages 384-389, 2007.

[3] S. Bingulac and R. Krtolica. On admissibility of pseudoobservability and pseudocontrollability indexes. IEEE Trans. Automat. Control, 32(10):920-922, October 1987.

[4] B. Dravie, P. Guillot, and G. Millérioux. Flatness and structural analysis as a constructive framework for private communication. Nonlinear Analysis: Hybrid Systems, 30:92-105, 2018.

[5] M. Fliess, J. Lévine, P. Martin, and P. Rouchon. A Lie-Bäcklund approach equivalence and flatness of nonlinear systems. IEEE Trans. Automat. Control, 44(5):922937, 1999.

[6] K. Fritzsche, Y. Guo, and K. Röbenack. Nonlinear control of non-observable nonflat mimo state space systems using flat inputs. Proc. of the 23rd International Conference on System Theory, Control and Computing (ICSTCC), pages 173-179. IEEE, 2019.

[7] K. Fritzsche and K. Röbenack. On the computation of differentially flat inputs. Proc. of the 22nd ICSTCC, pages 12-19. IEEE, 2018.

[8] K. Fritzsche and K. Röbenack. On a generalized flat input definition and physical realizability. Proc. of the 21st IFAC World Congress. IFAC, 2020.

[9] J.-P. Gauthier, H. Hammouri, and S. Othman. A simple observer for nonlinear systems with applications to bioreactors. IEEE Trans. Automat. Control, 37(6):875880, 1992.

[10] K. Graichen, V. Hagenmeyer, and M. Zeitz. A new approach to inversion-based feedforward control design for nonlinear systems. Automatica, 41(12):2033 - 2041, 2005.

[11] H. Hammouri and J. De Leon Morales. Observer synthesis for state-affine systems. Proc. 29th IEEE CDC, pages 784-785 vol. 2, 1990.

[12] Vagn Lundsgaard Hansen. Integrably parallelizable manifolds. Proceedings of the American Mathematical Society, 35(2):543-546, 1972.

[13] R. Hermann and A. J. Krener. Nonlinear controllability and observability. IEEE Trans. Automat. Control, 22(5):728-740, 1977.

[14] A.J. Krener and W. Respondek. Nonlinear observers with linearizable error dynamics. SIAM J. Control Optim., 23(2):197-216, 1985.

[15] C. Letellier and J.-P. Barbot. Flatness for an optimal control of chaotic systems using a minimal numbers of sensors and actuators. Submitted for publication, 2021. 
[16] J. Lévine. Analysis and Control of Nonlinear Systems: A Flatness-Based Approach. Springer, 2009.

[17] E. N. Lorenz. Deterministic nonperiodic flow. Journal of the Atmospheric Sciences, 20(2):130-141, 1963.

[18] F. Nicolau and W. Respondek. Flatness of multi-input control-affine systems linearizable via one-fold prolongation. SIAM J. Control Optim., 55(5):3171-3203, 2017.

[19] F. Nicolau, W. Respondek, and J.-P. Barbot. Constructing flat inputs for two-output systems. Proc. MTNS, pages 414-421, 2018.

[20] F. Nicolau, W. Respondek, and J.-P. Barbot. Flat inputs: theory and applications. SIAM J. Control Optim., 58(6):3293-3321, 2020.

[21] F. Nicolau, W. Respondek, J.-P. Barbot, and A. Ouslimani. Secure communication with the help of flat inputs for chaotic systems. IFAC-PapersOnLine, 51(33):109 - 114, 2018. 5th IFAC Conference on Analysis and Control of Chaotic Systems CHAOS 2018.

[22] P.J. Olver. Applications of Lie groups to differential equations, volume 107. SpringerVerlag New York, 1986.

[23] W. Respondek. Geometry of static and dynamic feedback. Lecture Notes at the Summer School on Mathematical Control Theory, Trieste, Italy, September 2001 and Bedlewo-Warsaw, Poland, September 2002.

[24] W. Respondek. Right and left invertibility of nonlinear control systems. H.J. Sussmann (ed.). Nonlinear Controllability and Optimal Control, pages 133-176. Routledge New York, 1990.

[25] W. Respondek. Symmetries and minimal flat outputs of nonlinear control systems. volume LNCIS 295 of New Trends in Nonlinear Dynamics and Control and their Applications, pages 65-86. Springer, 2003.

[26] O.E. Rössler. An equation for continuous chaos. Phys. Let. A, 57(5):397-398, 1976.

[27] R. Schenkendorf and M. Mangold. Parameter identification for ordinary and delay differential equations by using flat inputs. Theoretical Foundations of Chemical Engineering, 48(5):594-607, 2014.

[28] J. Stumper, F. Svaricek, and R. Kennel. Trajectory tracking control with flat inputs and a dynamic compensator. Proc. of the 2009 European Control Conference (ECC), pages 248-253, 2009.

[29] I.A. Tall. Flow-box theorem and beyond. Afr. Diaspora J. Math. (N.S.), 11(1):75102, 2011.

[30] P.V. Tan, G. Millérioux, and J. Daafouz. Invertibility, flatness and identifiability of switched linear dynamical systems: an application to secure communications. Proc of the 47th IEEE CDC'08, pages 959-964, 2008.

[31] P.V. Tan, G. Millérioux, and J. Daafouz. Left invertibility, flatness and identifiability of switched linear dynamical systems: a framework for cryptographic applications. International Journal of Control, 83(1):145-153, 2010.

[32] S. Waldherr and M. Zeitz. Conditions for the existence of a flat input. Internat. J. Control, 81(3):439-443, 2008.

[33] S. Waldherr and M. Zeitz. Flat inputs in the mimo case. IFAC Proceedings Volumes, 43(14):695-700, 2010.

[34] X.H. Xia and W.B. Gao. Non-linear observer design by observer canonical forms. Internat. J. Control, 47(4):1081-1100, 1988. 